\title{
Cohomological twisting of 2-linearization and extended TQFT
}

\author{
Jeffrey C. Morton
}

Received: 31 October 2012 / Accepted: 8 July 2013 / Published online: 6 August 2013

(C) Tbilisi Centre for Mathematical Sciences 2013

\begin{abstract}
In this paper, we describe a relation between a categorical quantization construction, called "2-linearization", and extended topological quantum field theory (ETQFT). We then describe an extension of the 2-linearization process which incorporates cohomological twisting. The 2-linearization process assigns 2 -vector spaces to (finite) groupoids, functors between them to spans of groupoids, and natural transformations to spans between these. By applying this to groupoids which represent the (discrete) moduli spaces for topological gauge theory with finite group $G$, the ETQFT obtained is the untwisted Dijkgraaf-Witten (DW) model associated to $G$. This illustrates the factorization of TQFT into "classical field theory" valued in groupoids, and "quantization functors", which has been described by Freed, Hopkins, Lurie and Teleman. We then describe how to extend this to the full DW model, by using a generalization of the symmetric monoidal bicategory of groupoids and spans which incorporates cocycles. We give a generalization of the 2-linearization functor which acts on groupoids and spans which have associated cohomological data. We show how the 3-cocycle $\omega$ on the classifying space $B G$ which appears in the action for the DW model induces a classical field theory valued in this bicategory.
\end{abstract}

Keywords TQFT $\cdot$ Groupoids $\cdot$ 2-Vector spaces $\cdot$ Gauge theory

Mathematics Subject Classification (1991) $18 \mathrm{E} 10 \cdot 20 \mathrm{~L} 05 \cdot 57 \mathrm{R} 56$

Communicated by Justin Roberts.

J. C. Morton ( $\varangle)$

Fakultät für Mathematik, Informatik und Naturwissenschaften, Fachbereich Mathematik,

Universität Hamburg, Bundesstraße 55, 20146 Hamburg, Germany

e-mail: Jeffrey.Morton@uni-hamburg.de 


\section{Introduction}

This paper demonstrates a construction of an extended topological quantum field theory (ETQFT), associated to any finite group $G$, together with an element of the group cohomology, $\omega \in H_{\text {grp }}^{3}\left(G, U(1)\right.$. The ETQFT $Z_{G}$ defined here is related to a topological gauge theory with finite gauge group $G$, and in particular to the DijkgraafWitten (DW) model based on $(G, \omega)$.

This construction involves two parts. The first part is a 2 -functor $\Lambda$, called "2-linearization", described by the author in [39]. At the level of objects, the 2 -functor $\Lambda$ takes finite groupoids to 2-vector spaces. A "twisted" form of $\Lambda$ is introduced here, which extends this to a larger 2-category in which the groupoids carry some cohomological data. The second part is a functor which, to any space, assigns the groupoid of flat $G$-bundles with connection. The cohomological data is derived from $\omega$.

There are three main purposes for this paper. First, we show how $\Lambda$ gives a categorical framework in which to understand some well-known constructions. Second, we see how these constructions give a physically-motivated interpretation of $\Lambda$. Third, we draw on this motivation to see how to generalize $\Lambda$ to the more physically meaningful "twisted" case.

Specifically, we first see how $\Lambda$ can be used to reconstruct the untwisted case of the 3D TQFT constructed originally by Dijkgraaf and Witten [13], and further developed by Freed and Quinn [17], as well as elsewhere. The characterization $\Lambda$ in terms of ambi-adjoint functors in [39] gives a conceptual account of various normalization factors which appear in the standard construction, and organizes the physical structure into a very general structure. In particular, it shows that the DW model factors into two parts: first, a "classical field theory" valued in a bicategory $\operatorname{Span}(\mathbf{G p d})$ whose objects are groupoids and whose morphisms are "span" diagrams; second, a "quantization functor" $\Lambda$.

We are led to a physical interpretation of the 2-linearization functor $\Lambda$ as a categorification of the path integrals (in the discrete case, "sums over histories") which are used in the construction of the DW model. For this reason, and following the terminology of Freed, Hopkins, Lurie, and Teleman [16], we often also refer to it as a "quantization functor".

The application to this model leads naturally to a generalization of $\Lambda$. Although $\Lambda$ is a canonical choice for quantization of $\operatorname{Span}(\mathbf{G p d})$, it is unable to express even full generality of the DW model, itself toy model of a quantum field theory. In particular, the general DW model is "twisted" by the group cocycle $\omega$ mentioned above, which gives a nontrivial topological action term. The cocycle is supplied by the classical part of the twisted theory and can be described in the language of bundles and gerbes [49]. The cocycle leads to a Lagrangian "action functional", which is a function valued in $U(1)$, and is constant in the untwisted case.

Extending to this case leads to a larger category $\operatorname{Span}(\mathbf{G p d})^{U(1)}$ of groupoids and spans equipped with cohomological data, and to a modification of the quantization functor, $\Lambda^{U(1)}$, which acts on this larger category. 
This is an example of the program of Freed [15] on the use of higher-algebraic structures for quantization, further developed in the elegant higher-algebraic framework by Freed et al. [16], in which there are two ingredients to an extended TQFT:

(1) A "classical" field theory, where the values of the fields live in an $n$-groupoid.

(2) A "quantization functor" which takes the $n$-groupoids to $(n+1)$-algebras and spans to morphisms of all degrees.

That work refers to "canonical quantization for classical field theories valued in $n$ groupoids". We do not follow this in full generality - in particular, to reproduce the DW model, we need consider only the situation down to codimension 2, unlike the full program of [16]. In particular, our classical field theory takes values in ordinary groupoids (rather than the more general setting of $n$-groupoids), equipped with some extra data.

The main result of this paper is a construction of an extended Topological Quantum Field Theory as a symmetric monoidal 2-functor from a certain bicategory of double cobordisms into 2-vector spaces, which reproduces the twisted DW model, in the general case of surfaces with boundary, as described by Freed and Quinn [17]. The key ingredients are $\mathcal{A}_{0}(-)_{G}^{\omega}$, given in Definition 10 , and $\Lambda^{U(1)}$, given in Definition 12). The main theorem is:

Theorem 5: Given a finite gauge group $G$ and 3-cocycle $\omega \in Z^{3}(B G, U(1))$, the symmetric monoidal 2-functor

$$
Z_{G}^{\omega}=\Lambda^{U(1)} \circ \mathcal{A}_{0}(-)_{G}^{\omega}: \mathbf{3} \mathbf{C o b}_{2} \rightarrow \mathbf{2 V e c t}
$$

reproduces the DW model with twisting cocycle $\omega$.

This factorization raises the possibility of applying the same quantization functor to other examples. The untwisted $\Lambda$ is a natural extension of the Baez-Dolan program of "groupoidification" (see, e.g. [5,6,39]), in which the category Span (Gpd) appears in many different contexts. The twisted variant $\Lambda^{U(1)}$ extends this further, and we expect that it should give generalizations of many such examples.

The plan of the paper is as follows: in Sect. 2 we recall the categorical setup of extended topological quantum field theories; in Sect. 3 we describe the untwisted form of the construction using 2-linearization as the quantization functor and show that it reproduces the untwisted DW model; in Sect. 4 we give some calculations to explicitly show some of the invariants computed by this process; in Sect. 5 we describe the cocycle-twisted variations of the gauge theory and the 2-linearization functor and show that these reproduce the twisted DW model; finally we offer some concluding remarks.

\section{Topological quantum field theories}

Here we summarize the context of TQFT and ETQFT in which we will be working, in categorical language. We will assume in the following a basic familiarity with 2categories, and refer the reader to works such as those by Cheng and Lauda [10], or Lack [30]. For a good introduction on higher category theory in the context of TQFTs, see work of Baez and Dolan [4]. 
We recall some background on the DW model, to give more context for the remarks above. Atiyah's axioms for an $n$-dimensional TQFT describe it as a symmetric monoidal functor

$$
Z: \mathbf{n C o b} \rightarrow \text { Hilb }
$$

where $\mathbf{n C o b}$ is a category whose objects are $(n-1)$-manifolds and whose morphisms are cobordisms. In general, a " $k$-tuply extended TQFT" assigns higher-categorical structures called $k$-vector spaces to manifolds of codimension $k$. In particular, it is a (weak, monoidal) $k$-functor:

$$
Z: \mathbf{n C o b}_{\mathbf{k}} \rightarrow \mathbf{k}-\text { Vect }
$$

(The relevance of 2-vector spaces to the setting of topology as in ETQFT has been described in more detail, for example, by Yetter [50].) In this paper we are only interested in the case $k=2$, though the construction given here might be generalized to higher $k$.

A topological quantum field theory (TQFT) is understood physically as a quantum field theory with no local degrees of freedom. In particular, we are interested in TQFTs given by gauge theories. Fields in gauge theory are connections on bundles over some base space. We assume such connections are invariant under one-parameter families of diffeomorphisms, that is, are flat. Thus, the only interesting information about them is given by holonomies around non-contractible loops.

\subsection{The category $\mathbf{n C o b}$}

In general, a TQFT can be described as a functor from a category of manifolds and cobordisms into vector spaces (or Hilbert spaces) and linear maps. A survey of categorical aspects of TQFT was given by Bartlett [7]. For our purposes, we first need to understand the category of cobordisms involved here.

A cobordism between compact manifolds $S_{1}, S_{2}$ is a compact manifold with boundary, $M$, with $\partial M$ isomorphic to the disjoint union $S_{1} \coprod S_{2}$. Cobordisms are composed by identifying their boundaries. For our purposes, it will be useful to think of cobordisms as special kinds of cospans in a category of manifolds with boundary, so that this is a special case of the composition of cospans by pushout. Our aim here is to describe a generalization of categories of cobordisms.

The bicategory $\mathbf{n} \mathbf{C o b}_{2}$ has:

- Objects: Compact $(n-2)$-manifolds $P$ (without boundary)

- Morphisms: Cobordisms $P_{1} \times I \stackrel{i_{1}}{\rightarrow} S \stackrel{i_{2}}{\leftarrow} P_{2} \times I$ where $S$ is an $(n-1)$-dimensional collared cobordism

- The 2-morphisms of $\mathbf{n} \mathbf{C o b}_{2}$ are generated by $n$-dimensional collared cobordisms with corners $M$, up to diffeomorphisms which preserve the boundary

Composition is by gluing along collars, which are included to ensure that there is a smooth structure on composites. 
Remark 1 In the following, all manifolds are compact, whether or not this is explicitly mentioned.

In [38] there is a definition of a bicategory $\mathbf{n C o b}_{2}$, as a sub-bicategory of $\operatorname{Cosp}^{2}$ (ManCorn), where ManCorn is the category of manifolds with corners. In fact, the definition given in [38] (as a "double bicategory") is slightly trickier than what we will use to define our TQFT. In particular, it is a cubical 2-category, which distinguishes between different classes 2-morphisms. Specifically, cobordisms of cobordisms are represented as squares, which we think of as describing "evolution of manifolds with boundary" in which the boundary need not be fixed. If the source and target are the horizontal edges of a square, the changing boundary would then be represented by $(n-1)$-dimensional cobordisms thought of as the vertical edges. Collar-fixing diffeomorphisms of cobordisms, on the other hand, are "bigons" with a single source and target 1-morphism. When we collapse to a bicategory, this distinction can be ignored, since any diffeomorphism has a corresponding "mapping cylinder" cobordism.

For our purposes, it is enough to use, as shown in [38], the bicategory obtained from the double bicategory described there, which is equivalent to the usual definition of $\mathbf{n} \mathbf{C o b}_{2}$. However, we want to emphasize here that treating the cobordism category explicitly as a category of cospans means that the "classical field theory" functor which we construct is really just induced by a functor on ManCorn. Specifically, it is induced by the contravariant functor $\mathcal{A}_{0}(-)_{G}$, which is simple to construct, and when applied to cospans of manifolds with corners, is easily seen to give spans of groupoids.

Details about smooth structure can be largely passed over here. Indeed, for our construction to work, it is only necessary to get an $n$-functor from the cobordism category into the appropriate form of $\operatorname{Span}(\mathbf{G p d})$. Since this is done, here by passing through the fundamental groupoid, only the homotopy types of the manifolds and cobordisms are detected by these invariants. Thus, the precise details of composing cobordisms with collars is not crucial for these ETQFTs based on gauge theory. It may be relevant for other field theories, however. TQFT is a special situation, which we now recall.

\subsection{TQFT and ETQFT as functors}

Atiyah's axiomatic formulation [2] of the axioms for a TQFT can be summarized as follows:

Definition 1 An $n$-dimensional Topological Quantum Field Theory is a (symmetric) monoidal functor

$$
Z: \mathbf{n C o b} \rightarrow \text { Vect }
$$

where nCob is the monoidal category of $(n-1)$-dimensional manifolds and $n$-dimensional cobordisms, and Vect is the monoidal category whose objects are vector spaces, whose arrows are linear transformations, and whose monoidal operation is the usual tensor product $\otimes$. 
A more general characterization of cobordism categories is the Baez-Dolan Cobordism Hypothesis, characterizing the $n$-category whose objects are points and whose $n$-morphisms are $n$-dimensional cobordisms (necessarily with corners). The characterization is that this category is a free symmetric monoidal $n$-category "with duals" in a suitable sense (details can be found in [4]). This has been proved by Lurie (see [33]). Christopher Schommer-Pries has given a presentation for $\mathbf{2} \mathbf{C o b}_{\mathbf{2}}$ as a symmetric monoidal bicategory [43], given in terms of Morse theory and a classification of singularities.

This takes us to ETQFTs, which are defined not just on manifolds with boundary, but also on manifolds with corners. This idea was introduced by Ruth Lawrence [32], under the name " $r$-ETFT", replacing the concept of vector space with that of $r$-vector space, Just as a TQFT assigns a space of states to a manifold and a linear map to a cobordisms, a (doubly) extended TQFT will assign some such algebraic data to manifolds of dimension $(n-r)$, and cobordisms up to dimension $n$. Our construction here will describe the situation where $r=2$.

Definition 2 The 2-category 2 Vect has:

- Objects: Finite-dimensional Kapranov-Voevodsky 2-vector spaces (i.e. $\mathbb{C}$-linear, finitely semisimple abelian categories)

- Morphisms: 2-linear maps (i.e. $\mathbb{C}$-linear functors, which are necessarily additive)

- 2-Morphisms: Natural transformations

This is a slight abstraction of the definition given in [26]. This category has a natural symmetric monoidal structure, using the Deligne tensor product of categories.

A straightforward categorification of Atiyah's description of a TQFT as a functor, as proposed by Lawrence, runs as follows:

Definition 3 An extended TQFT is a (symmetric monoidal) weak 2-functor

$$
Z: \mathbf{n C o b}_{2} \rightarrow \mathbf{2 V e c t}
$$

In particular, such a $Z$ assigns:

- To an $(n-2)$-manifold, a 2-vector space

- To an $(n-1)$-manifold, a 2-linear map between 2-vector spaces

- To an $n$-manifold, a 2-natural transformation between 2-linear maps

All this data satisfies the conditions for a weak 2-functor (e.g. it preserves composition and units up to coherent isomorphism, and so forth). The monoidal structure in 2Vect is the Deligne tensor product on abelian categories (see e.g. section 4.3 of [29]).

As before, a fuller version of this theory will use $\mathbf{2 H i l b}$ (see [3]) in place of 2Vect, but we will mostly omit this complication here.

\subsection{Topological Gauge theory and TQFTs}

Quantum field theories are often derived from classical field theories described in terms of gauge theory. In the topological case, a class of TQFTs and ETQFTs may 
be constructed from topological gauge theory, in which fields are flat connections on a manifold $B$. Being flat, the nontrivial information about a connection depends only on the topology of $B$.

In particular, all the information available about any connection comes in the form of holonomies of the connection around loops. The holonomy is an element $A(\gamma) \in G$ associated to a loop $\gamma$ in $B$, defining the "parallel transport" around that loop. The $G$-connection is flat exactly if the holonomy assigned to a loop depends only on the homotopy class of $\gamma$. To say this more conveniently, we first recall the definition (see Brown [9]):

Definition 4 The fundamental groupoid $\Pi_{1}(B)$ of a space $B$ is a groupoid with points of $B$ as its objects, and whose morphisms from $x$ to $y$ are just all homotopy classes of paths in $B$ starting at $x$ and ending at $y$.

Suppose $G$ is a group, understood as a one-object groupoid whose composition is the group multiplication. Then we have:

Definition 5 A flat $G$-connection is a functor

$$
A: \Pi_{1}(B) \rightarrow G
$$

A gauge transformation $\alpha: A \rightarrow A^{\prime}$ from one connection to another is a natural transformation of functors so that $\alpha_{x} \in G$ satisfies $\alpha_{y} A(\gamma)=A^{\prime}(\gamma) \alpha_{x}$ for each path $\gamma: x \rightarrow y$. Flat connections and natural transformations form the objects and morphisms of the groupoid of flat connections

$$
\mathcal{A}_{0}(B)_{G}=\left[\Pi_{1}(B), G\right]
$$

The groupoid of flat connections functor is the contravariant functor:

$$
\mathcal{A}_{0}(-)_{G}: \text { ManCorn } \rightarrow \text { Gpd }
$$

which, to $(X \stackrel{f}{\rightarrow} Y) \in$ ManCorn, assigns the restriction map $f^{*}$, precomposition with $f$.

(Here we are using the notation that $\left[C_{1}, C_{2}\right]$ is the category whose objects are functors from $C_{1}$ to $C_{2}$ and whose morphisms are natural transformations.)

In Sect. 3.3 we will see how this extends to a functor on $\operatorname{Cosp}^{2}$ (ManCorn), and therefore to $\mathbf{n} \mathbf{C o b}_{2}$, which takes values in a bicategory of groupoids and spans.

Thurston [46] showed that this groupoid of connections is equivalent, in the categorical sense, to the usual definition in terms of flat principal $G$-bundles. This is because, as categories, $G \simeq \mathbf{G}$-Tor, the category of $G$-torsors (sets with a $G$-action which are isomorphic as $G$-sets to $G$ itself). A flat connection on a principal $G$-bundle gives the fiber-selecting functor from $\Pi_{1}(B)$ to G-Tor, where the holonomy along a path transports each fiber. It is enough to use the groupoid of connections, since we are only interested in these constructions up to equivalence, so any representative of the equivalence class. 
(We note that, more precisely, the configuration spaces should be seen as stacks, which are determined by Morita equivalence classes of groupoids, and consequently everything we construct here is unchanged, up to equivalence, by taking Morita equivalent groupoids everywhere. This refinement is important for topological groupoids, but here we need not be concerned with it, since Morita equivalence and categorical equivalence are the same for finite groupoids.)

In gauge theory, two connections which are related by a gauge transformation describe physically indistinguishable states - the differences they detect are due only to the system of measurement used. The groupoid then describes the internal symmetry of a "physical" space of states. Now, rewriting Definition 5, if $\gamma: x \rightarrow x$ in $\Pi_{1}(B)$ is a loop, and $A$ and $A^{\prime}$ are two connections related by a gauge transformation $\alpha$, the relation between $A$ and $A^{\prime}$ can be expressed $A^{\prime}(\gamma)=\alpha(x)^{-1} A(\gamma) \alpha(x)$, that is, the holonomies assigned by the two connections around the loop are conjugate. So physically distinct holonomies correspond to conjugacy classes in $G$.

Indeed any category is equivalent, as a category, to its skeleton, so in general $\Pi_{1}(B) \cong \coprod_{b \in \pi_{0}(B)} \pi_{1}(B, b)$. The gauge transformations for connections measured from a fixed base point $b$ are determined by a single group element at $b$, acting on holonomies around any loop by conjugation. The groupoid $\mathcal{A}_{0}(B)_{G}$, which is the configuration space for our theory, is the "weak quotient" of the space $F u n\left(\pi_{1}(B), G\right)$ of connections by the action of gauge transformations at each base-point.

Proposition 1 For any compact manifold B (possibly with corners), and finite group $G$, the groupoid $\mathcal{A}_{0}(B)_{G}$ is essentially finite (equivalent as a category to a finite groupoid).

Proof To begin with, note that the functor category $\mathcal{A}_{0}(B)_{G}$ is indeed a groupoid, since any natural transformation $g$ assigns to a point $b \in B$ a group element $g_{b}$, which is invertible. The transformation $g^{-1}$ with $g_{b}^{-1}=\left(g_{b}\right)^{-1}$ is the inverse.

Next, note that for any space $B$,

$$
\Pi_{1}(B) \equiv \coprod_{i=1}^{n}\left(\pi_{1}\left(B_{i}\right)\right)
$$

The sum is taken over all path components of $B$. That is, objects in $\Pi_{1}(B)$ are by definition isomorphic if and only if they are in the same path component. The automorphisms for an object corresponding to path component $B_{i}$ are the equivalence classes of paths from any chosen point to itself-namely, $\pi_{1}\left(B_{i}\right)$. If $B$ is a compact manifold, so is each component $B_{i}$, which is also connected. But the fundamental group for a compact, connected manifold is finitely generated. So in particular, each $\pi_{1}\left(B_{i}\right)$ is finitely generated, and there are a finite number of components. So $\Pi_{1}(B)$ is an essentially finitely generated groupoid.

But if $\Pi_{1}(B)$ is essentially finitely generated, then since $G$ is a finite group, $\mathcal{A}_{0}(B)_{G}$ is an essentially finite groupoid. This is because each functor's object map is determined by the images of the generators, and there are finitely many such assignments. Similarly, $\Pi_{1}(B)$ is equivalent to its skeleton, and a natural transformation in this case is just given by a group element in $G$ for each component of $B$, so there are finitely many. 
We have described how to associated the groupoid $\mathcal{A}_{0}(B)_{G}$ to any manifold $B$. Next we will see how to use this to construct extended topological quantum field theories.

\section{ETQFT by 2-linearization}

Here we want to consider an explicit construction of a class of extended TQFTs based on a finite group $G$, using the groupoids of connections described in the previous section.

In [39], we defined a weak 2-functor $\Lambda$ from spans of groupoids to 2 -vector spaces. In particular, the construction we give here works by associating spans of groupoids to cobordisms, and then applying this $\Lambda$. These groupoids arise from the moduli space of flat connections on the source and target manifolds, as well as on the cobordism itself. These are connected by natural restriction maps to give spans. A similar line of reasoning gives spans of span maps associated to cobordisms between cobordisms.

We recap the key ingredient $\Lambda$ next.

\subsection{Groupoidification and 2-linearization}

Groupoidification is a term for the (non-systematic) process of finding an inverse to the (systematic) “degroupoidification" functor, which gives representations of Span (Gpd) in Vect, or Hilb. The goal is to find structures in $\operatorname{Span}(G p d)$ whose representations reproduce some chosen structure in Vect or Hilb. The reader may find more details on this program in a review by Baez et al. [5], and in Hoffnung's work on geometric representation theory [24]. The author has described an example of an application to physics, and in particular the combinatorics of Feynman diagrams in [37].

2-Linearization: the 2-functor $\Lambda$ gives a representation of $\operatorname{Span}(\mathbf{G p d})$ in 2-vector spaces. This is discussed in the general setting in [39].

Both invariants rely on different forms of a ubiquitous pull-push process, the best-known example of which is ordinary matrix multiplication. Indeed the relation to matrix multiplication is exactly the basis for the degroupoidification functor. In the context of the 2-linearization functor $\Lambda$, the "pull" and "push" refer to the direct and inverse limits of Vect-presheaves along a functor. This fundamental construction appears in many categories, notably toposes [34]. For abelian sheaves this is described in some generality by Kashiwara and Schapira [27]. The situation most closely relevant to the present case occurs in the setting of representations of rings [8].

For our immediate purposes, we can omit many of these considerations, but note that the ambidextrous (i.e. both left and right) adjunction between direct and inverse image functors valued in Vect gives us the extra structure used to construct $\Lambda$. This ambidextrous adjunction appears, indirectly, because a finite-dimensional vector space is canonically isomorphic to its double-dual. (For this reason, in infinite-dimensional situations, one properly ought to use Hilb-valued functors, which may be seen as equivariant Hilbert bundles. For the finite case, Vect is sufficient.) 


\subsection{The 2-linearization functor $\Lambda$}

The category $\operatorname{Rep}(X)$ of finite dimensional representations of a groupoid $X$ is the category of $X$-actions on bundles of vector spaces over the objects of $X$. In the essentially finite, discrete context we are working in, this just the same as the category of functors from $X$ into Vect, denoted [ $X$, Vect]. Such a representation category is a 2-vector space (i.e. a Vect-enriched abelian category).

The construction of $\Lambda$ uses the fact that, for any functor $f: X \rightarrow Y$ of essentially finite groupoids, there is an adjunction

$$
\operatorname{Rep}(X) \underset{f^{*}}{\stackrel{f_{*}}{\gtrless}} \operatorname{Rep}(Y)
$$

We describe these as $f^{*}$ ("pull") and $f_{*}$ ("push") between the 2-vector spaces of functors $[X$, Vect $]$ and $\left[Y\right.$, Vect]. In fact, this adjunction is "ambidextrous": $f_{*}$ is both a left and a right adjoint to $f^{*}$. The importance of ambidextrous adjunctions is discussed by Lauda [31] from the algebraic point of view which relates 2D TQFTs to Frobenius algebras.

The effect of $\Lambda$ on 2-morphisms can also be thought of in terms of a "pull-push" process, but here we use the unit and counit from the two adjunctions between $f^{*}$ and $f_{*}$. In particular, we use the unit from the adjunction where $f_{*}$ is a right adjoint, and the counit from the adjunction where it is a left adjoint. We denote the unit:

$$
\eta_{R}: \operatorname{Id}_{[X, \text { Vect }]} \Longrightarrow f_{*} f^{*}
$$

The counit is similarly denoted:

$$
\epsilon_{L}: f_{*} f^{*} \Longrightarrow \operatorname{Id}_{[X, \text { Vect }]}
$$

Remark 2 We note here that these two operations are a special case of the general "six-operation" framework [36]: in algebraic geometry, for a map $f: X \rightarrow Y$ of varieties (or schemes), one gets functors $f^{*}, f_{*}, f^{!}$and $f$ ! between categories of sheaves $\operatorname{Sh}(X)$ and $\operatorname{Sh}(Y)$. This is a special case, since we take our groupoids to have discrete topology, so all functors (as presheaves) are sheaves. Furthermore, $f^{*}$ has in the general case a different left adjoint $f_{*}$ and right adjoint $f_{!}$. However, in this case, the two adjoint pairs of functors coincide. This is due, indirectly, to the fact that objects in Vect are canonically isomorphic to their double-duals, as can be seen by the matrix representation of these 2-linear maps.

One way to summarize the structure we get uses a certain bicategory of spans of groupoids:

Definition 6 The symmetric monoidal bicategory $\operatorname{Span}(\mathbf{G p d})$ has:

- Objects: Essentially finite groupoids

- Morphisms: Spans of groupoids

- 2-Morphisms: Equivalence classes of spans of span maps 
The monoidal operation for $\operatorname{Span}(\mathbf{G p d})$ is determined by the fact that, on objects, it is the product in $\mathbf{G p d}$.

This generalizes a construction of a bicategory whose morphisms are spans, and whose 2-morphisms are span maps. In fact, Span (Gpd) as we have presented it might be better understood a 3-category. In general, the span construction on any bicategory will yield a (monoidal) tricategory, where the 3-morphisms are maps of spans of span maps, as described by Hoffnung [25]. Reducing to 3-isomorphism classes gives exactly the 2-morphisms described here, and makes our $\operatorname{Span}(\mathbf{G p d})$ a (symmetric) monoidal bicategory. We have chosen the current approach because of the up-to-diffeomorphism definition of 2-morphisms in $\mathbf{n} \mathbf{C o b}_{2}$.

For a category $\mathbf{C}$ with pullbacks, the $\operatorname{Span}(\mathbf{C})$ has many useful properties due to certain universal properties of the span construction [12] (for bicategories, a similar analysis is done in [28]). For example, taking categories of spans ensures that every morphism has a "dual" (the same span, considered with the opposite orientation), and is a minimal expansion of $\mathbf{C}$ with this property. The point of the following construction is to take these "duals" and represent them as ambi-adjoint functors.

Thus, it was shown [39] that the following defines a 2-functor:

Definition 7 The weak 2-functor

$$
\Lambda: \operatorname{Span}(\mathbf{G p d}) \rightarrow \mathbf{2 V e c t}
$$

assigns:

- For $X$ an essentially finite groupoid, the functor category $\Lambda X=[X$, Vect $]$

- For a span of groupoids $A \stackrel{s}{\leftarrow} X \stackrel{t}{\rightarrow} B$ in $\operatorname{Span}(\mathbf{G p d})$, the 2-linear map:

$$
\Lambda X=t_{*} \circ s^{*}: \Lambda A \longrightarrow \Lambda B
$$

- For a span between spans, $Y: X_{1} \rightarrow X_{2}$ for $X_{1}, X_{2}: A \rightarrow B$, as in:

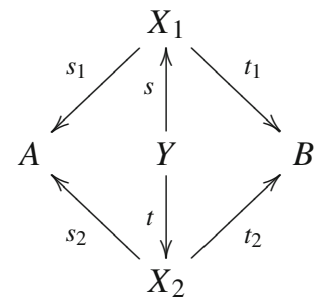

the natural transformation

$$
\Lambda(Y)=\epsilon_{L, t} \circ N \circ \eta_{R, s}:\left(t_{1}\right)_{*} s_{1}^{*} \Longrightarrow\left(t_{2}\right)_{*} s_{2}^{*}
$$

where $\epsilon_{L, t}$ is the counit for the left adjunction associated to $t$, and $\eta_{R, s}$ is the unit for the right adjunction associated to $s$, and $N$ is the Nakayama isomorphism between the left and right adjoints. 
We note that $\Lambda$ is a weak 2-functor, so there are also natural isomorphisms called the "compositor"

$$
\beta: \Lambda\left(X^{\prime} \circ X\right) \rightarrow \Lambda\left(X^{\prime}\right) \circ \Lambda(X)
$$

for each composable pair of spans $X$ and $X^{\prime}$, and the "unitors"

$$
U_{B}: 1_{\Lambda(B)} \stackrel{\sim}{\rightarrow} \Lambda\left(1_{B}\right)
$$

for each groupoid $X$. These are described in [39] in detail. So, briefly, is the case where the 2-morphism diagram is only required to commute up to isomorphism. These issues will not be required in the current context.

The role of the Nakayama isomorphism here is also described in more detail in [39], but is relevant here, so we will briefly recap this. In general, given a map $f: X \rightarrow Y$, there will be both a left and a right adjoint to $f^{*}$, the pullback of (in this case, Vectvalued) functors from $Y$ to $X$. These may be described in terms of the internal hom and $\otimes$ in Vect.

In each case, these "pushforwards" of a functor $F: X \rightarrow$ Vect to $Y$ will be described as a direct sum over all objects $x$ in the essential preimage of $y \in Y$. Since $F(x)$ gives a representation of $A u t(x)$, the summands are the induced representations along the associated homomorphism $\hat{F}: A u t(x) \rightarrow A u t(y)$. For the left adjoint, this is $\mathbb{C}[A u t(y)] \otimes_{\mathbb{C}[A u t(x)]} F(x)$ (a representation of $A u t(y)$ ), and for the right adjoint it is $\operatorname{hom}_{\mathbb{C}[\operatorname{Aut}(x)]}(\mathbb{C}[\operatorname{Aut}(y)], F(x))$ (that is, the hom-space as $\mathbb{C}[\operatorname{Aut}(x)]$-modules). The Nakayama isomorphism turns a map in the right adjoint (hom-space) to a vector in the left adjoint (tensor product) by the "exterior trace", averaging over a group action:

$$
\phi \mapsto \frac{1}{\# \operatorname{Aut}(x)} \sum_{g \in A u t(y)} g^{-1} \otimes \phi(g)
$$

This gives the natural transformations associated to 2-morphisms by 2-linearization. Note that we sum over $\operatorname{Aut}(y)$, which projects to the $A u t(y)$-invariant subspace, a canonical representative of a vector in the tensor product, but the "average" is given by dividing by the size of $A u t(x)$. This reflects the fact that we are pushing forward a representation of $\operatorname{Aut}(x)$, and it is necessary to make this an isomorphism when we are dealing with modules in general, say over $\mathbb{Z}$, rather than $\mathbb{C}$-vector spaces. In this setting, it merely sets a canonical scale, which turns out to reproduce the groupoid cardinality which appears in the groupoidification process of Baez and Dolan (see e.g. [6]).

The 2-linearization construction relies on the fact that having both covariant and contravariant functors $(-)^{*}$ and $(-)_{*}$ amounts to the same thing as having a single functor from Span(Gpd). In general, pairs of functors like this satisfying some nice properties are Mackey functors (see [21,40]). The situation is in general somewhat more complicated when groupoids are thought of as having topological spaces, rather than discrete sets, of objects and morphisms. However, we take advantage of the simplifying fact for the discrete case to construct an ETQFT for a discrete gauge group $G$. We describe this in the next section. 


\subsection{From cobordisms to spans}

In this section, we show the functoriality of the classical field theory part of the factorization $Z_{G}=\Lambda \circ \mathcal{A}_{0}(-)_{G}$.

Theorem 1 There is a 2-functor:

$$
\mathcal{A}_{0}(-)_{G}: \mathbf{n} \mathbf{C o b}_{2} \rightarrow \operatorname{Span}(\mathbf{G p d})
$$

\section{induced by the groupoid of flat connections functor of Definition 5}

Proof This will follow from the fact that there is an inclusion $\mathbf{n} \mathbf{C o b}_{2} \rightarrow \operatorname{Cosp}_{2}$ (ManCorn), the bicategory derived from $\operatorname{Cosp}^{2}$ (ManCorn) as in [38].

A cobordism in $\mathbf{n} \mathbf{C o b}_{2}$ can be seen as a particular 1-morphism (cospan) in the bicategory $\operatorname{Cosp}_{2}$ (ManCorn), given by inclusion maps:

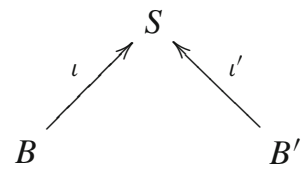

(Note that as described in [38], there are "collars" associated with these inclusions, but these have no effect on our construction so we shall ignore them here.)

Given two cobordisms $S: B_{1} \rightarrow B_{2}$ and $S^{\prime}: B_{2} \rightarrow B_{3}$, the composite $S^{\prime} \circ S$ is a (homotopy) pushout of two cospans (over $B_{2}$ ). The functor $\Pi_{1}$ also gives cospans of the fundamental groupoids, whose composite $\Pi_{1}\left(S^{\prime}\right) \circ \Pi_{1}(S)$ is a (weak) pushout. Then it is a well-known consequence of Brown's [9] groupoid version of the Van Kampen theorem that $\Pi_{1}\left(S^{\prime} \circ S\right) \simeq \Pi_{1}\left(S^{\prime}\right) \circ \Pi_{1}(S)$ (see also [23]).

In the next step, we apply $[-, G]: \operatorname{Cosp}(\mathbf{G p d}) \rightarrow \operatorname{Span}(\mathbf{G p d})$. So at this stage of the construction we have a span. To see that this operation is compatible with composition of cobordisms the essential fact is that the contravariant functor $[-, G]$ takes weak (homotopy) pushouts to weak pullbacks.

The composition of morphisms is by weak pushout of (collared) cospans in ManCorn. This still holds when we take fundamental groupoids. Applying $[-, G]$, we get spans of groupoids. Thus the corresponding diagram of spans contains a (weak) pullback square. Denoting the pull-backs along the inclusions by $p_{i}$ and $p_{i}^{\prime}$, we have this diagram:

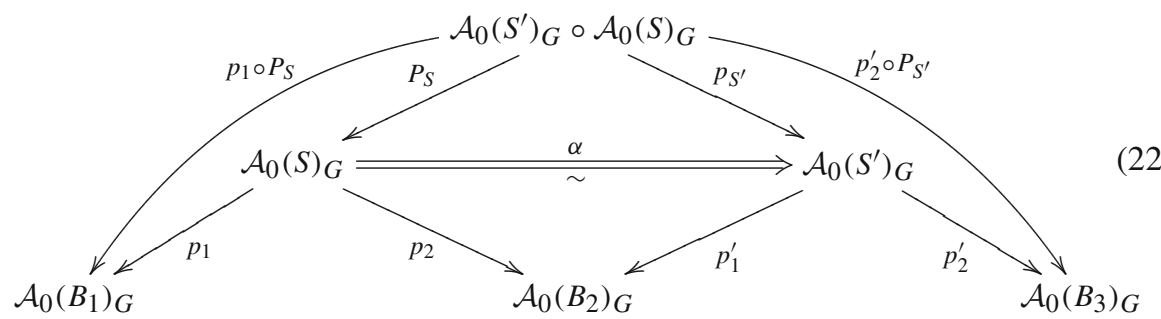


The weak pullback $\mathcal{A}_{0}\left(S^{\prime}\right)_{G} \circ \mathcal{A}_{0}(S)_{G}$ is canonically described (up to equivalence) as a comma category, whose objects are triples $A, f, A^{\prime}$ where $A \in \mathcal{A}_{0}(S)_{G}, A^{\prime} \in$ $\mathcal{A}_{0}\left(S^{\prime}\right)_{G}$, and $f: p_{2}(A) \rightarrow p_{1}^{\prime}\left(A^{\prime}\right)$. That is, for connections $A$ and $A^{\prime}$ on $S$ and $S^{\prime}$, the restrictions to $B_{2}$ are gauge equivalent, but not necessarily equal. Each different gauge equivalence gives a different object of $\mathcal{A}_{0}\left(S^{\prime}\right)_{G} \circ \mathcal{A}_{0}(S)_{G}$.

Thus, $\mathcal{A}_{0}\left(S^{\prime}\right)_{G} \circ \mathcal{A}_{0}(S)_{G} \simeq \mathcal{A}_{0}\left(S^{\prime} \circ S\right)_{G}$, where composition of cobordisms is by the (homotopy) pushout along the collared inclusions of the boundary $B_{2}$.

A similar argument shows the unitor property for $\mathcal{A}_{0}(-)_{G}$.

Finally, for 2-morphisms, we note that here, composition is by strict pullback and pushout since spans of spans are taken only up to isomorphism. Otherwise the same argument holds. Thus, we have a 2-functor into $\operatorname{Span}(\mathbf{G p d})$.

Remark 3 We also note here that a similar construction to the functor $[-, G]$ used here plays a role in a construction by Grandis [20] of TQFT via spans of sets. That construction works with topological spaces, rather than ManCorn, and $[-, S]$ then denotes the functor which assigns to a space $A$ the set of homotopy classes of maps from $A$ into $S$. That is, here we are concerned with the homotopy 1-type (a groupoid), rather than a 0-type (a set) of the space of maps into $S$. The result we need is shown in the general case of spaces by Chorny [11], and the groupoid case follows since groupoids are homotopy 1-types of spaces.

\subsection{Extended TQFT via $\Lambda$}

We can now describe explicitly how our ETQFT is constructed:

Definition 8 For any finite group $G$, define the 2-functor

$$
Z_{G}=\Lambda \circ \mathcal{A}_{0}(-)_{G}: \mathbf{n C o b}_{2} \rightarrow \mathbf{2 V e c t}
$$

Proposition 2 This $Z_{G}$ is an Extended TQFT.

Proof Since both $\mathcal{A}_{0}(-)_{G}$ and $\Lambda$ are weak, symmetric monoidal 2-functors, so is the composite $Z_{G}$, so this is indeed an Extended TQFT.

In particular, since by Proposition $2.3 \mathcal{A}_{0}(B)_{G}$ is an essentially finite groupoid, the main theorem of [39] then implies $\left[\mathcal{A}_{0}(B)_{G}\right.$, Vect] is a Kapranov-Voevodsky 2-vector space.

Remark 4 To describe it explicitly, given a finite group $G$, the extended TQFT $Z_{G}$ makes the following assignments:

- For a closed compact manifold $B, Z_{G}$ assigns the 2-vector space:

$$
Z_{G}(B)=\left[\mathcal{A}_{0}(B)_{G}, \text { Vect }\right]
$$

- For a cobordism between manifolds:

$$
B \stackrel{i}{\rightarrow} S \stackrel{i^{\prime}}{\leftarrow} B^{\prime}
$$


the weak 2-functor assigns a 2-linear map:

$$
Z_{G}(S)=\left(p^{\prime}\right)_{*} \circ p^{*}
$$

where $p$ and $p^{\prime}$ are the projections for the span of groupoids associated to $S$ by $\mathcal{A}_{0}(-)_{G}$.

- For a cobordism with corners between two cobordisms with the same source and target:

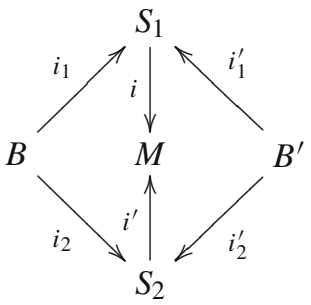

the natural transformation (16) becomes:

$$
\Lambda(M)=\epsilon_{L, p^{\prime}} \circ N \circ \eta_{R, p}:\left(p_{1}^{\prime}\right)_{*} \circ p_{1}^{*} \Longrightarrow\left(p_{2}^{\prime}\right)_{*} \circ p_{2}^{*}
$$

where $p^{\prime}$ and $p$ are as above. The coherence isomorphisms which make $Z_{G}$ a weak 2 -functor are those defined by $\Lambda$ as in [39]. (These appear in coordinates as matrices whose components are linear maps between the coefficients of the 2-linear maps).

\subsection{The 3D untwisted Dijkgraaf-Witten model as ETQFT}

Now we will consider the three-dimensional case in particular. Given a gauge group $G$, the DW model [13] is a topological gauge theory, involving flat $G$-connections on manifolds. For Lie groups, this theory is related to the Chern-Simons theory, but our interest here is for finite groups. The general theory of Lie groups can be understood from finite groups and simply connected Lie groups. This is because, as described by [13], the finite groups occur in exact sequences as either the group of components, or the fundamental group, of Lie groups (which can thus be used to reduce a general Lie group first to a connected, then a simply-connected, one).

TQFTs equivalent to the DW model are often defined as invariants for triangulated manifolds, found by considering compatible $G$-colorings of the (directed) edges. This is done, for example, by Yetter [51], and in discussion in the chapter on TQFT of the unpublished notes by Porter [42], which also discuss an extension to categorical groups. Yetter showed that one can obtain an invariant of manifolds which is independent of triangulation via a colimit over all triangulations.

While triangulations are crucial in the case of categorical groups, for ordinary groups, the $G$-colorings of edges in a triangulation amount to flat $G$-connections. These can be described in terms of maps in $\operatorname{Hom}\left(\pi_{1}(M), G\right)$, or equivalently in 
$\operatorname{Hom}(M, B G)$, where $B G$ is the classifying space of $G$. We use the former description here, since the groupoid structure is easiest to see in that form.

Note that the normalizing factors which appear in the 2-functor $\Lambda$ as the Nakayama isomorphism (19) count the size of automorphism groups of objects. In the mapping space $\operatorname{Hom}(M, B G)$, these appear as the size of homotopy groups of connected components (as in the "homotopy order" as described in [42]). The homotopy order of a connected space $X$ with base-point $x$ and only finitely many nontrivial homotopy groups is:

$$
\#^{\pi}(X, x)=\prod_{i=1}^{\infty}\left|\pi_{i}(X, x)^{(-1)^{i}}\right|
$$

(In the case of $B G$, and $\operatorname{Hom}(M, B G)$, all homotopy groups for $i \geq 2$ are trivial, so this reproduces the groupoid cardinality.)

We now consider explicitly how the DW model and a natural ETQFT extension of it can be found from the 2-functor $Z_{G}$ defined in this paper.

Recall that the category $\mathbf{2} \mathbf{C o b}$ occurs in $\mathbf{3} \mathbf{C o b}_{2}$ as the category of automorphisms of the object $\emptyset$, which is particularly interesting since $\varnothing$ is the monoidal unit in $\mathbf{3} \mathbf{C o b}_{2}$. We can ask about the effect of $Z_{G}$ restricted to this automorphism category. It turns out to be just the same as the DW model in 3 dimensions.

Our main theorem is the following:

Theorem 2 There is a natural isomorphism between the functor $Z_{G \mid \text { Aut }(\emptyset)}$ and the untwisted DW model.

Proof We need to exhibit the natural equivalence at the level of objects and morphisms.

Now, $Z_{G}(\emptyset) \cong$ Vect, whose single basis 2 -vector (mapped to $\mathbb{C}$ under the equivalence) is the trivial representation of the trivial group.

So a cobordism in Aut (Ø) gives a 2-linear map from Vect to Vect, which is naturally equivalent to giving a vector space (and in particular, a complex vector space with a specified basis, and thus a Hilbert space). Cobordisms in Aut (Ø) are 2-dimensional cobordisms from $\emptyset$ to $\emptyset$, or in other words, closed 2-dimensional manifolds. These are, up to diffeomorphism, just genus- $g$ tori $\Sigma_{g}$.

Given $\Sigma_{g}$, the DW model produces a $d$-dimensional Hilbert space $\mathcal{H}_{\Sigma_{g}}$, where

$$
\begin{aligned}
d & =\left|V_{g}\right| \\
& =\left|\operatorname{hom}\left(\pi_{1}\left(\Sigma_{g}\right), G\right) / G\right|
\end{aligned}
$$

with a basis canonically indexed by conjugacy classes of flat connections $\gamma \in$ $\operatorname{hom}\left(\pi_{1}\left(\Sigma_{g}\right), G\right) / G$.

Now, thinking of $\Sigma_{g}$ as a cobordism, that is, as a cospan:

$$
\emptyset \rightarrow \Sigma_{g} \leftarrow \varnothing
$$

we get the span of groupoids

$$
! \stackrel{!}{\leftarrow} \mathcal{A}_{0}\left(\Sigma_{g}\right)_{G} / / G \stackrel{!}{\rightarrow} \mathbf{1}
$$


Here, the map ! denotes the unique map into the terminal object, which we name in order to express the following formulas. By the above, we find that $Z_{G}\left(\Sigma_{g}\right)$ : $Z_{G}(\emptyset) \rightarrow Z_{G}(\emptyset)$ can be represented as a $1 \times 1$ matrix, with

$$
\begin{aligned}
Z_{G}\left(\Sigma_{g}\right) \mathbb{C}, \mathbb{C} & =\left\langle!^{*}(\mathbb{C}), !^{*}(\mathbb{C})\right\rangle \\
& =\bigoplus_{\gamma \in \mathcal{A}_{0}\left(\Sigma_{g}\right)_{G} / G} \operatorname{hom}(\mathbb{C}, \mathbb{C}) \\
& \cong \bigoplus_{\gamma \in \mathcal{A}_{0}\left(\Sigma_{g}\right)_{G} / G} \mathbb{C}
\end{aligned}
$$

This is canonically isomorphic to $\mathcal{H}_{\Sigma_{g}}$.

So suppose we have a 3-dimensional cobordism between 2-manifolds $\Sigma$ and $\Sigma^{\prime}$, which amounts to a cospan of cospan maps:

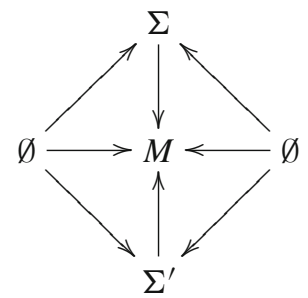

then again there is a span of span maps, where the central morphisms are the restrictions along the boundary inclusion maps:

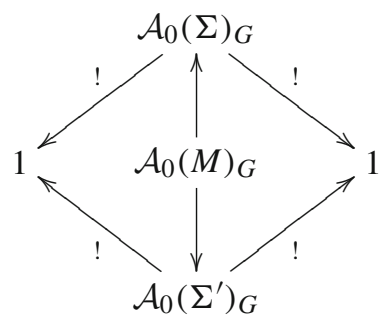

Now, this gives a 2-linear map $Z_{G}(M)$, and again there is only one entry, so we find

$$
Z_{G}(M)_{\mathbb{C}, \mathbb{C}}: Z_{G}(\Sigma)_{\mathbb{C}, \mathbb{C}} \rightarrow Z_{G}\left(\Sigma^{\prime}\right)_{\mathbb{C}, \mathbb{C}}
$$

And in particular, we can write the components of this linear map as:

$$
\begin{aligned}
\left(Z_{G}(M)_{\mathbb{C}, \mathbb{C})_{\gamma, \gamma^{\prime}}}\right. & =\left|\left(\pi, \pi^{\prime}\right)^{-1}\left([\gamma],\left[\gamma^{\prime}\right]\right)\right| \\
& =\sum_{\left[\gamma^{\prime \prime}\right] \in\left(\pi, \pi^{\prime}\right)^{-1}\left([\gamma],\left[\gamma^{\prime}\right]\right)} \frac{1}{\left|A u t\left(\gamma^{\prime \prime}\right)\right|}
\end{aligned}
$$




$$
=\sum_{\left[\gamma^{\prime \prime}\right]} \frac{\left|\left\{\gamma^{\prime \prime} \in\left[\gamma^{\prime \prime}\right]\right\}\right|}{|G|}
$$

In this last sum, we have only observed that the size of the automorphism group of a given connection $\gamma^{\prime \prime}$ is just the size of the full group divided by the size of the orbit of $\gamma^{\prime \prime}$, namely the equivalence class $\left[\gamma^{\prime \prime}\right]$. But now we can convert a sum over this equivalence class into a sum over all connections, and get:

$$
\left(Z_{G}(M)_{\mathbb{C}, \mathbb{C}}\right)_{\gamma, \gamma^{\prime}}=\frac{1}{|G|} \sum_{\gamma^{\prime \prime}} 1
$$

So this is the same linear map produced by the DW model.

The DW model itself is somewhat more general than what we have discussed so far. In fact, the 2-linearization framework used here constructs a particular ETQFT, which is the "untwisted" theory. Twisted DW models may be defined using a class $\alpha$ from the group cohomology of $G$. To produce twisted DW models, one must extend the 2-linearization framework to include cocycles. In the twisted DW model, the "topological action" associated to a given flat connection, is a unit complex number associated to that connection, determined by $\alpha$.

In Sect. 5 we will describe how to extend this result through a generalized 2linearization process.

\section{Example calculations}

Although the construction for an ETQFT will work in any dimension, its main features are visible in any dimension at least 2 , to allow codimension-2 submanifolds. Some calculations in low dimensions illustrate the invariants produced by the ETQFT.

\section{1 $Z_{G}$ on manifolds}

We can give the dimension of the 2-vector space assigned to any manifold by counting its basis objects, which yields the following straightforward fact:

Proposition 3 The $K V$ 2-vector space $Z_{G}(B)$ for any connected manifold $B$ has dimension:

$$
\sum_{[A] \in \mathcal{A} / G} \mid\{\text { irreps of } \operatorname{Aut}(A)\} \mid
$$

The sum is over equivalence classes of connections on $B$.

Proof This is just a special case of the general fact about representation categories for groupoids. 
The groupoid $\mathcal{A}_{0}(B)_{G}$ is equivalent to its skeleton $S$, whose objects are the gauge equivalence classes of connections on $B$. The morphisms are the stabilizer groups at each object. That is, they are exactly the gauge transformations which fix a representative of each class. Then $[S$, Vect $] \simeq\left[\mathcal{A}_{0}(B)_{G}\right.$, Vect $]$, but $[S$, Vect $]$ is a KV vector space, hence equivalent to some $\mathbf{V e c t}^{m}$, where $m$ is the number of non-isomorphic simple objects.

A functor $F: S \rightarrow$ Vect assigns a vector space to each object [ $A$ ] (equivalence class of connections), carrying a representation of $\operatorname{Aut}(A)$. Two functors giving inequivalent representations cannot have any nontrivial natural transformation between them, by Schur's lemma. On the other hand, any representation of $\operatorname{Aut}(A)$ is a direct sum of irreducible representations. So a choice of simple object in $\left[\mathcal{A}_{0}(B)_{G}\right.$, Vect $]$ amounts to a choice of $[A]$, and an irreducible representation of $\operatorname{Aut}(A)$. The statement follows immediately.

Our construction works, in principle, for manifolds of any dimension, though computations become more involved in higher dimension as one might expect. Next we consider some particular examples.

Example 1 All 1-dimensional manifolds are equivalent to a collection of circles, so to understand the effect on 1-manifolds, it suffices to know that the 2-vector space assigned to the circle $S^{1}$ by $Z_{G}$ is:

$$
\left[\mathcal{A}_{0}\left(S^{1}\right)_{G}, \text { Vect }\right]=\Lambda \circ \mathcal{A}_{0}\left(S^{1}\right)_{G}=\operatorname{Rep}\left(\mathcal{A}_{0}\left(S^{1}\right)_{G}\right)
$$

The fundamental group of the circle is $\mathbb{Z}$, and $\Pi_{1}\left(S^{1}\right)$ is thus equivalent to $\mathbb{Z}$ as a one-object category. A functor $g: \mathbb{Z} \rightarrow G$ is determined by $g(1) \in G$, which we also denote $g$ for convenience. A natural transformation is a conjugacy relation: $h: g \rightarrow g^{\prime}$ assigns to the single object in $\mathbb{Z}$ a morphism $h \in G$, and the naturality condition that $g^{\prime} h=h g$, or simply $g^{\prime}=h g h^{-1}$.

Thus, $\mathcal{A}_{0}\left(S^{1}\right)_{G}$ is equivalent to the groupoid whose objects are $g \in G$, and whose morphisms are conjugacy relations between elements. This is the transformation groupoid of the adjoint action of the group $G$ on itself, also known as $G$ "weakly modulo" $G$, or $G / / G$. This is also the discrete form of the loop groupoid $L G$, as summarized in the account by Willerton [49]).

Finally, the 2-vector space corresponding to the circle is the category of representations of $G / / G$.

$$
Z_{G}\left(S^{1}\right)=\operatorname{Rep}(G / / G)
$$

is generated by a basis of irreducible objects, the elements of which are labelled by pairs: a conjugacy class $[g]$ in $G$, and an irreducible representation of Aut $([g])$. All representations are direct sums of these. One can also think of an object of $Z_{g}\left(S^{1}\right)$ as a $G$-equivariant vector bundle on the groupoid of connections on $S^{1}$, which is just $G$.

The skeleton of $G / / G$ has as objects the conjugacy classes of $G$, and each object has $\operatorname{Aut}(g)<G$, the stabilizer subgroup of $g$, which is the centralizer $C_{g}$. 
Since a general 1-manifold is isomorphic to $S^{1} \cup \cdots \cup S^{1}$, the effect of $Z_{G}$ then just amounts to:

$$
\begin{aligned}
Z_{G}\left(S^{1} \cup \cdots \cup S^{1}\right) & =Z_{G}\left(S^{1}\right) \otimes \cdots \otimes Z_{G}\left(S^{1}\right) \\
& =\operatorname{Rep}(G / / G) \otimes \cdots \otimes \operatorname{Rep}(G / / G)
\end{aligned}
$$

(The monoidal operation $\otimes$ is the Deligne tensor product for Abelian categories. It is analogous to the tensor product for modules or vector spaces and is dual to the internal $H o m$ functor, so that $A \otimes B$ is a representing object for bi-2-linear functors out of $A \times B$.)

Let us briefly consider the case where objects are 2-dimensional manifolds (as in the 4D TQFT). We will not study the 4D ETQFT in detail, but this will illustrate that the same construction will work in that case.

Example 2 Consider the torus $T^{2}=S^{1} \times S^{1}$. We want to find

$$
Z_{G}\left(T^{2}\right)=\left[\mathcal{A}_{T^{2}}(B)_{G}, \text { Vect }\right]
$$

The category $\mathcal{A}_{0}\left(T^{2}\right)_{G}$ is again the category of functor $\Pi_{1}\left(T^{2}\right) \rightarrow G$ and natural transformations. The groupoid $\Pi_{1}\left(T^{2}\right)$ is equivalent to its skeleton, namely the fundamental group of $T^{2}$. This is just $\mathbb{Z}^{2}$. So the functor $F \in\left[\mathbb{Z}^{2}, G\right]$ is a group homomorphism from $\mathbb{Z}^{2}$ to $G$, and thus determined by the images of the two generators $(1,0)$ and $(0,1)$. Since $\mathbb{Z}^{2}$ is abelian, the images $g_{1}=F(1,0)$ and $g_{2}=F(0,1)$ must commute.

So the objects of $\mathcal{A}_{0}\left(T^{2}\right)_{G}$ are indexed by commuting pairs of elements $\left(g_{1}, g_{2}\right) \in$ $G^{2}$. (We note here that in the case of a topological group, this is a space of some interest in itself; see e.g. [1]. In the discrete case, this is simply a set.)

A natural transformation $g: F \rightarrow F^{\prime}$ assigns to the single object $\star$ of $\mathbb{Z}^{2}$ a morphism in $G$ - that is, a group element $h$. This must satisfy the naturality condition $h F(a) h^{-1}=F^{\prime}(a)$ for all $a$. This will be true for all $a$ in $\mathbb{Z}^{2}$ as long as it is true for $(1,0)$ and $(0,1)$.

In other words, for functors $F$ and $F^{\prime}$ represented by $\left(g_{1}, g_{2}\right) \in G^{2}$ and $\left(g_{1}^{\prime}, g_{2}^{\prime}\right) \in$ $G^{2}$, the natural transformations $\alpha: F \rightarrow F^{\prime}$ are represented by group elements $h \in G$ which act in both components at once, so $\left(h^{-1} g_{1} h, h^{-1} g_{2} h\right)=\left(g_{1}^{\prime}, g_{2}^{\prime}\right)$.

So we have that the groupoid $\mathcal{A}_{0}\left(T^{2}\right)_{G}$ is equivalent to $A / / G$, where $A=\left\{\left(g_{1}, g_{2}\right) \in\right.$ $\left.G^{2}: g_{1} g_{2}=g_{2} g_{1}\right\}$, and the action of $G$ on $A$ comes from the action on $G^{2}$ as above.

So the 2-vector space $Z_{G}\left(T^{2}\right)$ is just the category of Vect-presheaves on $A$, equivariant under the given action of $G$. This assigns a vector space to each connection $\left(g_{1}, g_{2}\right)$ on $T^{2}$, and an isomorphism of these vector spaces for each gauge transformation $h:\left(g_{1}, g_{2}\right) \mapsto\left(h^{-1} g_{1} h, h^{-1} g_{2} h\right)$. Equivalently (taking a skeleton of this), we could say it gives a vector space for each equivalence class $\left[\left(g_{1}, g_{2}\right)\right] \in G^{2}$ under simultaneous conjugation, and a representation of $G$ on this vector space.

A similar pattern will apply to a 2-dimensional surface of genus $k$. 


\section{2 $Z_{G}$ on cobordisms}

To help clarify the construction we have described, we consider some examples for particular cobordisms, and particular groups $G$.

We should emphasize here that although we are primarily considering the case $n=3$ in order to establish a link with the DW theory, we will express our results generically.

In particular, Turaev established [47] that a (2+1)-dimensional TQFT is determined by a modular tensor category (MTC) C (i.e. monoidal category with a modular structure). Such a category has a finite set of generators (the number of generators is the "rank" of the MTC), and the monoidal structure is determined by the matrices representing $Z_{G}(Y)$.

In the framework we have been describing, $\mathbf{C}=Z_{G}\left(S^{1}\right)$ is the 2-vector space for the circle. The monoidal structure for $\mathbf{C}$ as a MTC turns out to be given by the value of $Z_{G}$ on the "pair of pants" cobordism, or "trinion", $Y: S^{1} \sqcup S^{1} \rightarrow S^{1}$. This depicts two circles coalescing into one circle, by a cobordism which is topologically a sphere with the interiors of three disks removed. It therefore determines a monoidal structure by:

$$
Z_{G}(Y): \mathbf{C} \otimes \mathbf{C} \rightarrow \mathbf{C}
$$

Now, up to diffeomorphism, all 2-dimensional cobordisms can be written as composites of some number of copies of $Y$ and its dual, so knowing this monoidal structure completely determines the effect of the TQFT on morphisms. It is common to describe this using the monoidal structure directly.

However, our general framework can be applied to other values of $n$, for which there is no such simple "normal form" for cobordisms. However, we can still compute 2linear maps in these cases. It is common to describe a MTC C as a monoidal category, giving fusion rules for the "tensor product" functor $Z_{G} Y$. However, we prefer here simply to use a standard matrix representation of a 2-linear map, since these techniques would apply equally in higher dimensions where no such presentation exists, though of course when $n=3$ the two approaches are equivalent.

Example: The pair of pants Applying the monoidal functor $Z_{G}$ to the pair of pants gives a 2-linear map:

$$
Z_{G}(Y): Z_{G}\left(S^{1}\right) \otimes Z_{G}\left(S^{1}\right) \rightarrow Z_{G}\left(S^{1}\right)
$$

The basis of generating objects for the two 2-vector spaces are the irreducible representations of the corresponding groupoid. Given irreducibles $V$, and $W$, the coefficients of the 2-linear map $Z_{G}(Y)$ are, by Frobenius reciprocity (see [39]):

$$
Z_{G}(Y)_{V, W} \cong \operatorname{hom}_{R e p\left(\mathcal{A}_{0}(Y)_{G}\right)}\left(p_{1}^{*}(V), p_{2}^{*}(W)\right)
$$

That is, one pulls back the basis 2-vectors to give representations $p_{1}^{*}(V)$ and $p_{2}^{*}(W)$ of $\mathcal{A}_{0}(Y)_{G}$, the middle groupoid of the span. The coefficient is the "inner product"the internal hom, which is the space of intertwiners between the two pulled-back 
Fig. 1 Connection for pants

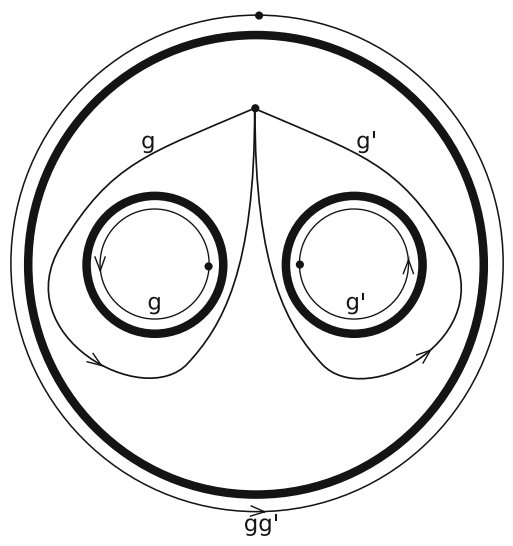

representations. By Frobenius reciprocity, this amounts to counting the multiplicities of each generating irreducible in the target 2-vector space in the image of the chosen generating irreducible in the source.

Since the fundamental groupoid of $S^{1} \cup S^{1}$ is just $\Pi_{1}\left(S^{1}\right) \cup \Pi_{1}\left(S^{1}\right)$, (a disjoint union of two copies of $\Pi_{1}\left(S^{1}\right) \cong \mathbb{Z}$ ), a functor into $G$ then amounts to two choices $g, g^{\prime} \in G$. A gauge transformation amounts to a conjugation by some $h \in G$ at each of two chosen base points, one in each component:

$$
\begin{aligned}
\mathcal{A}_{0}\left(S^{1} \cup S^{1}\right)_{G} & \cong(G \times G) / /(G \times G) \\
& \cong(G / / G)^{2}
\end{aligned}
$$

where $G \times G$ acts on itself by conjugation component-wise. This is illustrated in Fig. 1 . The connection on $Y$ has holonomies $g$ and $g^{\prime}$ around the two holes. On $S^{1} \cup S^{1}$, this restricts to a connection with holonomies $g$ and $g^{\prime}$ respectively, and on $S^{1}$ to the product (since the outside $S^{1}$ is homotopic to the composite of the two loops).

The fundamental groupoid is equivalent to $\pi_{1}(Y)=F\left(\gamma_{1}, \gamma_{2}\right)$, the free group on two generators. A functor in $\left[\Pi_{1}(Y), G\right]$ thus amounts to a pair of elements $\left(g, g^{\prime}\right)$, the images of the two generators. A gauge transformation amounts to conjugation at the single object (a chosen base point in $Y$ ). So we have the groupoid:

$$
\mathcal{A}_{0}(S)_{G} \cong(G \times G) / / G
$$

in which $G$ acts on $G \times G$ by conjugation in both components at once. Thus we have the span:

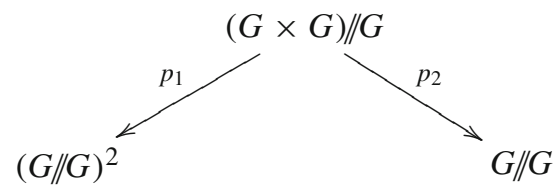


Both projections are restrictions of a connection on $Y$ to the corresponding connection on the components of the boundary. It is clear from the figure that on objects:

$$
\begin{aligned}
& p_{1}: \quad\left(g_{1}, g_{2}\right) \Rightarrow\left(g_{1}, g_{2}\right) \\
& p_{2}: \quad\left(g, g^{\prime}\right) \Rightarrow g g^{\prime}
\end{aligned}
$$

and on morphisms

$$
\begin{aligned}
& p_{1}:\left(h:\left(g_{1}, g_{2}\right) \rightarrow\left(h g_{1} g^{-1}, h g_{2} h^{-1}\right)\right) \Rightarrow\left((h, h):\left(g_{1}, g_{2}\right) \rightarrow\left(h g_{1} h^{-1}, h g_{2} h^{-1}\right)\right) \\
& p_{2}:\left(h:\left(g, g^{\prime}\right) \rightarrow\left(h g h^{-1}, h g^{\prime} h^{-1}\right)\right) \Rightarrow\left(h: g g^{\prime} \rightarrow h g g^{\prime} h^{-1}\right)
\end{aligned}
$$

The classes of connections on $Y$ are of the form $\left[\left(g, g^{\prime}\right)\right]$ for $g, g^{\prime} \in G$, and the class is an equivalence class modulo gauge transformations, conjugating by $(h, h)$. The classes for $S^{1} \cup S^{1}$ are of the form ([g], $\left.\left[g^{\prime}\right]\right)$, since equivalence is by conjugation by $\left(h, h^{\prime}\right) \in G^{2}$. So connections which are gauge equivalent on $S^{1} \cup S^{1}$ may be restrictions of inequivalent connections on $Y$.

Finally, suppose we have a functor $f: \mathcal{A}_{0}\left(S^{1} \cup S^{1}\right)_{G} \rightarrow$ Vect: that is, a representation of $\mathcal{A}_{0}\left(S^{1}\right)_{G} \times \mathcal{A}_{0}\left(S^{1}\right)_{G}$, and transport it by $Z_{G}(Y)=\left(p_{2}\right)_{*} \circ\left(p_{1}\right)^{*}$. That is, we first pull back along $p_{1}$ from $S^{1} \cup S^{1}$ to $Y$, then push forward along $p_{2}$ to $S^{1}$.

Now, an irreducible representation of $\mathcal{A}_{0}\left(S^{1}\right)_{G} \times \mathcal{A}_{0}\left(S^{1}\right)_{G}$ amounts to a pair of irreducible representations of $\mathcal{A}_{0}\left(S^{1}\right)_{G}$. Each one amounts to a choice of isomorphism class $[g]$ in $G / / G$, and a representation $V$ of $A u t(g)$. Call these $([g], V)$ and $\left(\left[g^{\prime}\right], V^{\prime}\right)$. Then the pair $\left([g],\left[g^{\prime}\right]\right)$ is the image of any $\left[\left(g, g^{\prime}\right)\right]$ in $(G \times G) / / G$ for some pair $\left(g, g^{\prime}\right)$ representing $\left([g],\left[g^{\prime}\right]\right)$. This will then be pushed down by $m$ to a representation of Aut $\left(g_{1}\right)$, where $g_{1}=g g^{\prime}$. There may be more than one $\left[\left(g, g^{\prime}\right)\right]$ for which this holds for a given $\left[g_{1}\right]$. In fact, following [39], we can then find that the image is:

$$
\left(\pi_{2}\right)_{*} \circ \pi_{1}^{*}\left(g_{1}\right) \cong \bigoplus_{\left(g, g^{\prime}\right) \in\left\{\left([g],\left[g^{\prime}\right]\right) \mid g g^{\prime}=g_{1}\right\}} \mathbb{C}\left[\operatorname{Aut}\left(g_{1}\right)\right] \otimes \mathbb{C}\left[\operatorname{Aut}\left(g, g^{\prime}\right)\right]\left(V \otimes V^{\prime}\right)
$$

where the direct sum is over all choices of $\left(g, g^{\prime}\right)$ representing $\left([g],\left[g^{\prime}\right]\right)$ and satisfying $g g^{\prime}=g_{1}$, up to equivalence in $(G \times G) / / G$. The action of $G$ on each component is as we have described. On morphisms, we get the direct sum of the isomorphisms between these copies of $\mathbb{C}$ (this formula is a special case of the general form for Kan extension in an enriched category-see e.g. [14]).

Now suppose $G$ is abelian. Then each element is in a unique conjugacy class. The irreducible representations of $G$ are 1-dimensional, forming $\hat{G}$, the character group of $G$. For abelian groups, this is just $\hat{G} \cong G$. So the simple representations are labelled by $G \times G$. This and general structure theorems for abelian groups make this case simple.

Lemma 1 If $G=G_{1} \oplus \cdots \oplus G_{n}$ is the direct sum of $n$ abelian groups, then $\mathcal{A}_{0}\left(S^{1}\right)_{G}$ is:

$$
G / / G \cong\left(G_{1} / / G_{1}\right) \times \cdots \times\left(G_{n} / / G_{n}\right)
$$


Table 1 Generators of $Z_{S_{3}}\left(S^{1}\right)$

\begin{tabular}{lll}
\hline Class $[g]$ & Group $\operatorname{Stab}(g)$ & \multicolumn{1}{l}{ Representations } \\
\hline$[\mathrm{h}]$ & & \\
& & \\
1 (identity) & $S_{3}$ & $\mathbb{C}, \Gamma=\square, \sigma$ \\
[t] (transposition) & $\mathbb{Z}_{2}=\{1, t\}$ & $\mathbb{C}$ and $\sigma$ \\
[s] (3-cycle) & $\mathbb{Z}_{3}=\left\{1, s, s^{2}\right\}$ & $\mathbb{C}, \phi, \phi^{2}$ \\
\hline
\end{tabular}

This follows directly from the definitions, and, combined with structure theorems for abelian groups and simple calculations for cyclic groups, gives the simple result:

Proposition 4 For $G$ a finite abelian group, the 2-linear map $Z_{G}(Y)$ is given by group operation + in $G \times G$ :

$$
Z_{G}(Y):\left(\left(g_{1}, g_{2}\right),\left(g_{1}^{\prime}, g_{2}^{\prime}\right)\right) \mapsto\left(g_{1}+g_{1}^{\prime}, g_{2}+g_{2}^{\prime}\right)
$$

The case of $G$ nonabelian is more interesting, since there may be more than one representative of $\left([g],\left[g^{\prime}\right]\right)$ contributing to a given term, and the stabilizer groups are different for different objects. Since the matrix form of 2-linear maps $Z_{G}(Y)$ are in general rather large even for fairly small $G$, so, we will here only illustrate certain interesting components in some simple cases, namely $G=S_{3}$ and $G=S_{4}$. Since in general these matrices are quite large, we will simply find a few blocks.

Example 3 First, find $Z_{S_{3}}\left(S^{1}\right)=\operatorname{Rep}\left(S_{3} / / S_{3}\right)$. As usual, this is generated by irreducible objects labelled by $([g], \rho)$, where $[g]$ is a conjugacy class in $G=S_{3}$, and $\rho$ is an irreducible representation of $\operatorname{Stab}(g) \subset G=S_{3}$. The groupoid $S_{3} / / S_{3}$ has stabilizer groups and irreducible representation as in Table 1 . For $[t]$ and $[s]$, the stabilizer groups are abelian, so for $\mathbb{Z}_{2}$ we have the trivial and sign representations, and for $[s]$ we have the irreducible representations of $\mathbb{Z}_{3}$ on $\mathbb{C}$ as before. For $S_{3}$, the three irreducible reps are labelled by three-block Young tableaux (see e.g. [45]), though these include the trivial representation $\mathbb{C}=\square \square$ and the sign representation $\sigma=\boxminus$. (The remaining irreducible representation, $\Gamma=\square$ is the 2-dimensional representation of $S_{3}$ given by the action of $S_{3}$ on the vertices of a triangle.)

Then $Z_{S_{3}}(Y): Z_{S_{3}}\left(S^{1} \coprod S^{1}\right) \rightarrow Z_{S_{3}}\left(S^{1}\right)$ is a 2-linear map taking representations of $\left(S_{3} / / S_{3}\right)^{2}$ to representations of $S_{3} / / S_{3}$, which are as described above. An irreducible representation of $\left(S_{3} / / S_{3}\right)^{2}$ is labelled by a pair $\left(([g], \rho),\left(\left[g^{\prime}\right], \rho^{\prime}\right)\right)$ of irreducible representations of $S_{3} / / S_{3}$.

The functor $Z_{S_{3}}(Y)$ can then be described by a $(64 \times 8)$ matrix of vector spaces. The entries are given by Frobenius reciprocity, pulling representations back along $m$ and $\Delta$ to representations of $\mathcal{A}_{0}(Y)_{G}=\left(S_{3} \times S_{3}\right) / / S_{3}$. So in particular, we get a sum over isomorphism classes in $\mathcal{A}_{0}(Y)_{G}$. These are given in Table 2.

Since both elements of a pair $\left(g_{1}, g_{2}\right)$ are conjugated by the same $g$ in this quotient action, we can distinguish cycles and permutations, as in $\left([s],\left[s^{2}\right]\right)$. Thus, there are two possible preimages of ( $[t],[t])$, depending on whether the two permutations $t$ and $t^{\prime}$ are the same, and similarly for $([s],[s])$. 
Table 2 Isomorphism classes of $\left(S_{3} \times S_{3}\right) / / S_{3}$

\begin{tabular}{clll}
\hline$\left[\left(g_{1}, g_{2}\right)\right]$ & $\operatorname{Stab}\left(\left[\left(g_{1}, g_{2}\right)\right]\right)$ & $\operatorname{Im}(m)$ & $\operatorname{Im}(\Delta)$ \\
\hline$[\mathrm{h}]$ & & & \\
{$[(1,1)]$} & $S_{3}$ & {$[1]$} & $([1],[1])$ \\
{$[(1, t)]$} & $\mathbb{Z}_{2}$ & {$[t]$} & $([1],[t])$ \\
{$[(1, s)]$} & $\mathbb{Z}_{3}$ & {$[s]$} & $([1],[s])$ \\
{$[(t, 1)]$} & $\mathbb{Z}_{2}$ & {$[t]$} & $([t],[1])$ \\
{$[(t, t)]$} & $\mathbb{Z}_{2}$ & {$[1]$} & $([t],[t])$ \\
{$\left[\left(t, t^{\prime}\right)\right]$} & 1 & {$[s]$} & $([t],[t])$ \\
{$[(t, s)]$} & 1 & {$[t]$} & $([t],[s])$ \\
{$[(s, 1)]$} & $\mathbb{Z}_{3}$ & {$[s]$} & $([s],[1])$ \\
{$[(s, t)]$} & 1 & {$[t]$} & $([s],[t])$ \\
{$[(s, s)]$} & $\mathbb{Z}_{3}$ & {$[s]$} & $([s],[s])$ \\
{$\left[\left(s, s^{2}\right)\right]$} & $\mathbb{Z}_{3}$ & {$[1]$} & $([s],[s])$ \\
\hline
\end{tabular}

So then we have that in matrix form $Z_{S_{3}}(Y)_{\left.([g], \rho),\left(\left[g_{1}\right], \rho_{1}\right),\left(\left[g_{2}\right], \rho_{2}\right)\right)}$ is given by a direct sum over the isomorphism classes in $\left(S_{3} \times S_{3}\right) / / S_{3}$ lying over $[g]$ by $m$ and over $\left(\left[g_{1}\right],\left[g_{2}\right]\right)$ by $\Delta$ (that is, non-conjugate pairs with $g_{2} g_{1}=g$ ). The coefficients for particular representations show the multiplicity of $\rho$ in the image of $\left(\rho_{1}, \rho_{2}\right)$. Unlike the abelian case, there are nontrivial coefficients from $([t],[t])$ to two different elements, $[s]$ and [1].

For example, we now find the block of $Z_{S_{3}}(Y)$ corresponding to the objects $([t],[t])$ and [1]. There is a single object in $\left(S_{3} \times S_{3}\right) / / S_{3}$ lying over these objects, namely $[(t, t)]$. Restricting to these objects, we have the span of automorphism groups:

$$
\mathbb{Z}_{2} \times \mathbb{Z}_{2} \stackrel{\Delta}{\leftarrow} \mathbb{Z}_{2} \stackrel{i}{\rightarrow} S_{3}
$$

(The map $i: \mathbb{Z}_{2} \rightarrow S_{3}$ is the injection homomorphism which takes the non-identity element of $\mathbb{Z}_{2}$ to $t \in S_{3}$.) We can calculate the block of $Z_{S_{3}}(Y)$ with indices given by the irreducible representations of these groups, namely $\{(\mathbb{C}, \mathbb{C}),(\mathbb{C}, \sigma),(\sigma, \mathbb{C}),(\sigma, \sigma)\}$, and $\{\mathbb{C}, \Gamma, \sigma\}$, respectively.

We find these by pulling back each representation to $\mathbb{Z}_{2}$ along $\Delta$ or $i$, and using Schur's lemma. One can easily find the block to be (using integers to represent vector spaces):

$$
Z_{\mathbb{S}_{3}}(Y)_{1,([t],[t])}=\left(\begin{array}{lll}
0 & 1 & 1 \\
1 & 1 & 0 \\
1 & 1 & 0 \\
0 & 1 & 1
\end{array}\right)
$$

In the example above, we see that the matrix form of $Z_{G}(Y)$ need not be a multiplication matrix for a group, as it is for the abelian case, essentially because the image of an irreducible may not itself be irreducible. However this example is still special in that only one object in the middle groupoid $G \times G / / G$ contributes to any given matrix 
entry. This is not true in general. The following (abbreviated) example illustrates the point in a more general framework than the above.

Example 4 If $G=S_{4}$, then $\mathcal{A}_{0}\left(S^{1}\right)_{G}=S_{4} / / S_{4}$ has isomorphism classes given by the conjugacy classes of permutations of 4 elements. These are classified by 4-box Young diagrams, of which there are five:

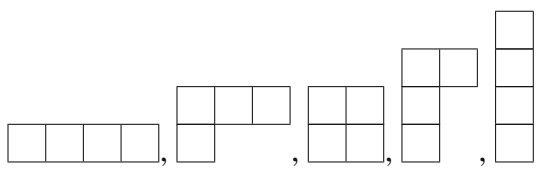

In the same way, $\mathcal{A}_{0}\left(S^{1} \cup S^{1}\right)_{G}$ has isomorphism classes given by pairs of such diagrams.

As in Example 3, we find a single block of $Z_{S_{4}}(Y)$, namely the block corresponding to ( $(\square, \square), \square \square$ ). Here we are using the diagram which corresponds to the conjugacy class of a 3-cycle (i.e. a permutation of four elements with one fixed point). In the usual cycle notation for permutations, this object is [(123)(4)].

The point now is that there are two classes in $\left(S_{4} \times S_{4}\right) / / S_{4}$ which project to $[((123)(4),(123)(4))]$ under $\Delta$ and [(123)(4)] under the multiplication map $m$. That is, there are two conjugacy classes of pairs of 3-cycles whose product is a 3-cycle. Representatives of these two classes are: $a=((123)(4),(123)(4))$, where the product is $m(a)=(132)(4)$; and $b=((123)(4),(243)(1))$. where the product is $m(b)=$ (143)(2). It is straightforward to check these are the only cases.

Now, Aut ((123)(4)) $\cong \mathbb{Z}_{3}$ : precisely the powers of this 3-cycle stabilize it under conjugation (4 must be a fixed point of any $\pi \in A u t((123)(4))$, and a transposition would change the cycle). So, since $a$ consists of two copies of this cycle, Aut $(a) \cong \mathbb{Z}_{3}$ also. On the other hand, Aut $(b)=\{I d\}$ : no permutation stabilizes both permutations in the pair $b$.

Thus, to find the ( $\left(\square \square, \square \square\right.$ ), $\square \square$ ) block of $Z_{S_{4}}(Y)$, we take a direct sum over $a$ and $b$ of the restriction-induction functors. These come from two spans of automorphism groups from $\mathbb{Z}_{3}^{2}$ to $\mathbb{Z}_{3}$. One can check that at $a$ we have the span:

$$
\mathbb{Z}_{3}^{2} \stackrel{\Delta}{\leftarrow} \mathbb{Z}_{3} \stackrel{i d}{\rightarrow} \mathbb{Z}_{3}
$$

The corresponding 2-linear map is just the multiplication map. On the other hand, at $b$ we have the span:

$$
\mathbb{Z}_{3}^{2} \stackrel{i}{\leftarrow}\{I d\} \stackrel{i}{\rightarrow} \mathbb{Z}_{3}
$$

In each case, the maps are the inclusions of the identity. Since the representations of $\mathbb{Z}_{3}$ (i.e. characters in $\hat{\mathbb{Z}}_{3}$ ) all pull back to the unique, trivial, representation of $\{I d\}$, Schur's lemma says the resulting matrix has $\mathbb{C}$ in each component. So taking the direct sum over $a$ and $b$, we find the block is: 


$$
\left(\begin{array}{lllllllll}
2 & 1 & 1 & 1 & 2 & 1 & 1 & 1 & 2 \\
1 & 2 & 1 & 1 & 1 & 2 & 2 & 1 & 1 \\
1 & 1 & 2 & 2 & 1 & 1 & 1 & 2 & 1
\end{array}\right)
$$

The other blocks are all found in a similar way (though we note that this is the only block for the case $G=S_{4}$ where more than one object appears in the direct sum.)

The final aspect of our weak 2-functor is its effect on 2-morphisms.

\section{3 $Z_{G}$ on cobordisms of cobordisms}

Now we consider the situation of a cobordism $M$ between cobordisms.

Recall that we are taking advantage of the construction [38] of a double bicategory of cobordisms $\mathbf{n} \mathbf{C o b}_{2}$, consistent with the cubical approach of [19,20]. This is a (weak) cubical 2-category, having horizontal and vertical 1-morphisms which are both cobordisms, and horizontal and vertical 2-morphisms which are diffeomorphisms between these. A square in $\mathbf{n} \mathbf{C o b}_{2}$ is a cobordism between cobordisms. We may intuitively understand the horizontal 1-morphisms at the top and bottom as the (vertical) source and target. The (horizontal) boundaries change, by the cobordisms which are the vertical 1-morphisms at the sides of the square. The situation is, of course, completely symmetric, so we may exchange the roles of horizontal and vertical in this interpretation.

The cubical picture is only a slightly more general picture than the more common bicategory view of cobordisms. The usual bicategory of cobordisms has as 2-morphisms which correspond exactly to those squares with horizontal boundaries constrained to be identity cobordisms (that is, the boundaries do not change). However, as discussed in [38], it is possible to reduce $\mathbf{n} \mathbf{C o b}_{2}$ to a bicategory, which is equivalent to the usual bicategory of cobordisms. This uses the fact that horizontal and vertical morphisms are both cobordisms, they can be composed with each other, and squares can be taken to 2-morphisms in the bicategory of cobordisms. The only information lost in this process is precisely how a given cobordism factors into horizontal and vertical parts.

In the construction we give here, in order to make the connection between the extended TQFT we construct and the DW model, we will use the bicategory 2 Vect as our target. Just because this is a bicategory, and because we want to make use of the quantization 2-functor $\Lambda$ from [39] we use this reduction of $\mathbf{n} \mathbf{C o b}_{2}$ to a bicategory.

In Fig. 2 we show an example, which can be construed as taking a pair of pants $Y$ to its reversed version $Y^{\dagger}$.

This clearly depicts a square in the double bicategory, since both source and target change in this process.

Our construction will then use the fact that there is an inclusion

$$
\mathbf{n C o b}_{2} \rightarrow \operatorname{Cosp}^{2}(\text { ManCorn })
$$

Here, a cobordism becomes a span of inclusion maps. Thus the (collarable) inclusions of these boundary components, and the corners, into this cobordism give the following 
Fig. 2 A 2-morphism in $\mathbf{3} \mathbf{C o b}_{2}$

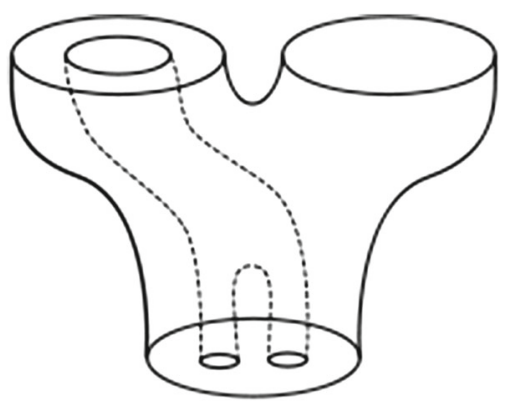

square in $\operatorname{Cosp}^{2}$ (ManCorn):

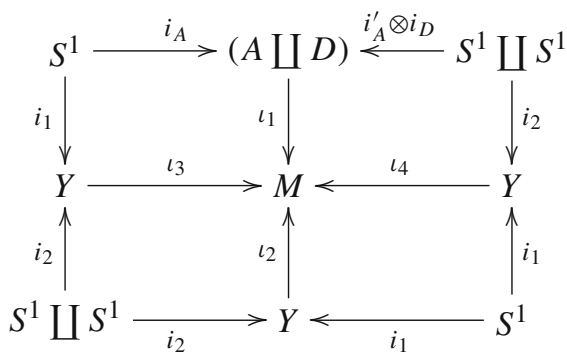

Here, $A$ is the annulus and $D$ the disk in the top horizontal cobordism, the $Y$ are instances of the pair of pants, and $M$ is the whole 3-dimensional manifold with corners. The leftmost vertical cospan is the inner cobordism, and the rightmost is the outer. The maps come from the obvious inclusions.

To get a corresponding 2-morphism in the bicategory $\mathbf{3} \mathbf{C o b}_{2}$, one must make some arbitrary choices, to choose the source and target objects from the four corners of the square. A corollary of this choice is that we lose the extra information encoded in the other two corners of the square, and the distinction between horizontal and vertical (hence change of boundary along the cobordism $M$ ).

The convention we adopt here is to take the source as the upper left and the target as the lower right. The source and target morphisms are then found by composing around the corners of the square. This convention turns $M$ into a cobordism between two cobordisms, each of which goes from $S^{1}$ to $S^{1}$. That is, $M$ becomes a cospan of cospan maps, forming a 2-morphism between the two cobordisms:

$$
Y \circ(A \coprod D) \rightarrow M \leftarrow Y \circ Y^{\dagger}
$$

as illustrated in Fig. 3. Here, $Y^{\dagger}$ is the adjoint of $Y$ as a cobordism, namely $Y$ with direction reversed. This step of the construction of $Z_{G}$ is slightly awkward since $\mathbf{n} \mathbf{C o b}_{2}$ is most generally a double bicategory, that is, a weak cubical 2-category, and 2Vect is most naturally a bicategory (that is, a weak globular 2-category). As mentioned in Sect. 2.1, this bicategory is equivalent to the more commonly used form of $\mathbf{n} \mathbf{C o b}_{2}$. 

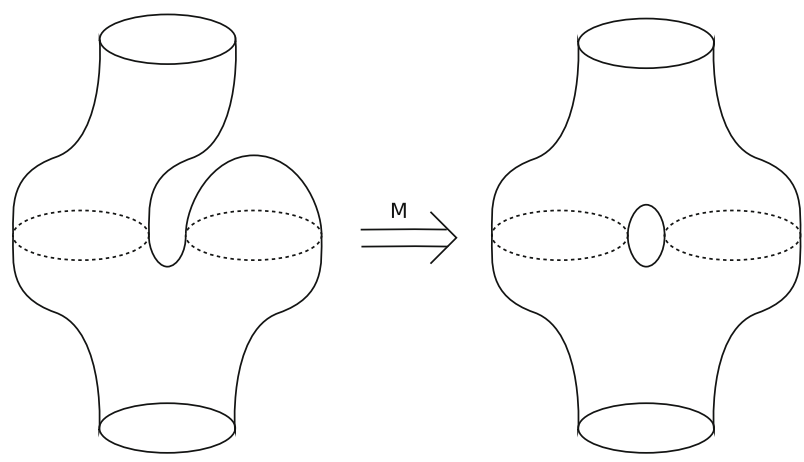

Fig. 3 The same 2-morphism, in a bicategory

We have chosen this method of reconciling them rather than the alternate approach of using some cubical version of 2 Vect for several reasons: cubical $n$-categories are simply less familiar, the (globular) bicategory 2 Vect is already in common use, and the quantization functor $\Lambda$ is adapted to it and has a straightforward relation to the groupoidification program.

The left-hand composite $S$ just amounts to the identity cobordism for $S^{1}$. So in particular, we can also regard this calculation as giving a cobordism from the identity cobordism on $S^{1}$ (the cylinder) to $Y \circ Y^{\dagger}$. The latter topologically a genus-1 surface with removed disks at the input and output boundaries. Applying $\mathcal{A}_{0}(-)_{G}$ gives a span of connection groupoids, all $G / / G$, with two identity maps. The right-hand cobordism is the composite $S^{\prime}=Y^{\dagger} \circ Y$.

Now, the point of using this inclusion of $\mathbf{n C o b}_{2}$ into $\operatorname{Cosp}^{2}$ (ManCorn) is that we can then extend the contravariant functor $\mathcal{A}_{0}(-)_{G}:$ ManCorn $\rightarrow$ Gpd to give a functor $\mathbf{n} \mathbf{C o b}_{2} \rightarrow \operatorname{Span}(\mathbf{G p d})$. Each object in a cospan then gives a groupoid, and the contravariant turns a cospan into a span. Likewise, for 2-morphisms, $\mathcal{A}_{0}(-)_{G}$ gives a span of spans of groupoids of connections, as in (15). We can then combine this

In the case where objects in $\mathbf{n} \mathbf{C o b}_{2}$ are empty manifolds $\emptyset$, cobordisms between two empty sets are themselves manifolds (with empty boundary), and cobordisms between these have boundary, but no nontrivial corners. So we have just a cobordism from one manifold to another. It is reasonable to expect that in this case, the extended TQFT based on a group $G$ should give results equivalent to those obtained from a TQFT based on the same group, suitably reinterpreted. This is indeed the case.

Example 5 Suppose we have two cobordisms $S$ and $S^{\prime}$ from $\emptyset$ to $\emptyset$, and a cobordism with (empty!) corners $M: S \rightarrow S^{\prime}$. In fact, $M$ should be thought of as a cobordism between manifolds, in a precisely analogous way that $Z_{G}(S)$ can be thought of as a TQFT giving a vector space for the manifold $S$.

In particular, we have

$$
Z_{G}(S) \cong\left(-\otimes \mathbb{C}^{k}\right)
$$

and 


$$
Z_{G}\left(S^{\prime}\right) \cong\left(-\otimes \mathbb{C}^{k^{\prime}}\right)
$$

The $k$ and $k^{\prime}$ are the number of isomorphism classes of connections on $S$ and $S^{\prime}$ respectively. If we think of these as being vector spaces $\mathbb{C}^{k}$ and $\mathbb{C}^{k^{\prime}}$ assigned by a TQFT, then a cobordism should assign a linear map $T: \mathbb{C}^{k} \rightarrow \mathbb{C}^{k^{\prime}}$. Indeed, such a linear map will give rise to a natural transformation from $Z_{G}(S)$ to $Z_{G}\left(S^{\prime}\right)$ by giving, for any objects $V \in$ Vect on the left side of the diagram, the map $1_{V} \otimes T$ on the right side. Moreover, all such natural transformations arise this way.

A cobordism between cobordisms gives rise to a natural transformation:

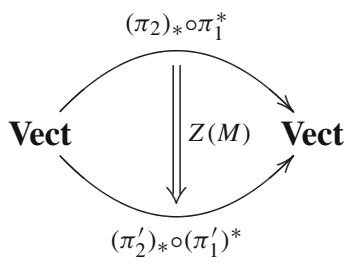

As discussed in [39], this reduces to the groupoidification functor

$$
D: \operatorname{Span}(\mathbf{G p d}) \rightarrow \text { Vect }
$$

and our construction just yields a TQFT. That is, each 2-linear map can then be described as a $1 \times 1$ matrix of vector spaces (that is, a vector space), and the natural transformations are just described in this one component by a single linear map.

Now we look, in the 3D case, at a more general 2-morphism $M$ in $\mathbf{n} \mathbf{C o b}_{2}$ and find $Z_{G}(M)$ for a general $G$.

Example 6 Next, consider the cobordism $M$ between cobordisms from Fig. 2, discussed above. The corresponding square (65).

To compute $Z_{G}(M)$, we should convert $M$ to a 2-morphism in the bicategory $\mathbf{3 C o b}_{2}$, then apply $Z_{G}$. However, consider first the effect of $\mathcal{A}_{0}(-)_{G}$ on (65), giving (up to equivalence) the following double span of groupoids:

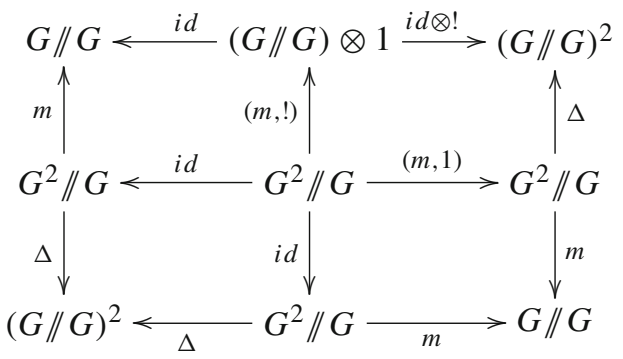

The corresponding 2-morphism in $\operatorname{Span}(\mathbf{G p d})$, can be found either by applying $\mathcal{A}_{0}(-)_{G}$ directly to the cospan of cospans obtained from (65) by composing around 
corners, or else by finding (71), and composing here. These are equivalent since $\mathcal{A}_{0}(-)_{G}$ is functorial.

In either case, we have the span of span maps:

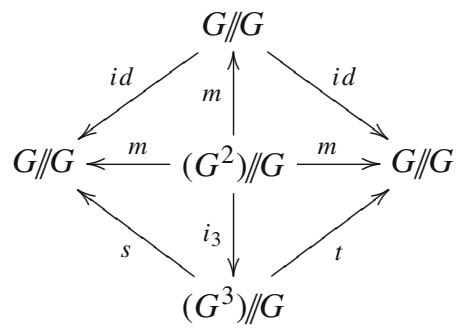

where the maps are given by:

$$
i_{3}:\left(g_{1}, g_{2}\right) \mapsto\left(g_{1}, g_{2}, 1\right)
$$

and

$$
s:\left(g_{1}, g_{2}, g_{3}\right) \mapsto g_{2}^{-1} g_{1}
$$

and

$$
t:\left(g_{1}, g_{2}, g_{3}\right) \mapsto g_{2}^{-1} g_{3} g_{1} g_{3}^{-1}
$$

We can then apply $\Lambda$ to find $Z_{G}(M)$. Again, by functoriality, we can either compose the 2-linear map for the pair of pants $Y$ and its dual, or apply $\Lambda$ to (72). Following [39], the natural transformation $Z_{G}(M): Z_{G}(S) \rightarrow Z_{G}\left(S^{\prime}\right)$ is in general:

$$
Z_{G}(M)=\epsilon_{L, i_{3}} \circ N \circ \eta_{R, m}
$$

For a connection $a$ on the source and representation $F$ of $G / / G$ (the central groupoid of the top span), these maps do the following to a vector $v$ in the representation space $F(a)$ :

$$
\eta_{R, m}(F)(a): v \mapsto \bigoplus_{[A] \mid m(y) \cong a} \frac{1}{\# \operatorname{Aut}(y)} \sum_{h \in A u t(a)} h^{-1} \otimes h(v)
$$

and

$$
\epsilon_{L, i_{3}}\left(F^{\prime}\right)\left(a^{\prime}\right): \bigoplus_{[A] \mid i_{3}(A) \cong a^{\prime}} h_{A} \otimes v \mapsto \sum_{[A] \mid i_{3}(A) \cong x} i_{3}\left(h_{A}\right) v
$$

(Objects $A$ are connections on $M$ ). So the composite gives: 


$$
Z_{G}(M)(F)(a)(v)=\bigoplus_{a^{\prime} \mid i_{3}(A) \cong a^{\prime}, m(A) \cong a} \frac{1}{\# A u t(A)} \sum_{[A] \mid i_{3}(A) \cong a^{\prime}} \sum_{h \in A u t(A)} i_{3}\left(h^{-1}\right) h(v)
$$

In particular, a connection $a \in G / / G$ is given by one group element $g \in G$, so this amounts to:

$$
Z_{G}(M)(F)(g)(v)=\bigoplus_{g^{\prime} \in G} \frac{1}{\# A u t\left(g^{\prime}, g^{\prime} g^{-1}\right)} \sum_{h \in A u t\left(g^{\prime}, g^{\prime} g^{-1}\right)} v
$$

Note that in this case, the only objects of $G^{3} / / G$ with nontrivial contribution are those of the form $\left(g^{\prime}, g^{\prime} g^{-1}, 1\right)$, and $i_{3}$ takes a gauge transformation represented by $h$ to one represented by the same $h$. Thus, $i_{3}\left(h^{-1}\right) h(v)=v$.

\section{Twisting and 2-linearization}

We now would like to see how our categorified quantization process $\Lambda: \operatorname{Span}($ Gpd $) \rightarrow$ 2 Vect generalizes to a twisted 2-linearization, $\Lambda^{U(1)}$. Then our main result generalizes to a claim that the twisted DW model is a composite of $\Lambda^{U(1)}$ and a "classical field theory" valued in groupoids equipped with such cocycles. These classical field theories are then classified by choices $(G, \omega)$, where $G$ is a finite group and $\omega \in H_{g r p}^{3}(G, U(1)$ is a cohomology class. The "quantization functor" $\Lambda^{U(1)}$ will be the same for all such ETQFTs.

Throughout this section, there will be several theorems which involve the construction of symmetric monoidal bicategories, and symmetric monoidal 2-functors (elsewhere often called symmetric monoidal homomorphisms) between them. We will not be completely explicit about every aspect of these claims.

The literature on symmetric monoidal bicategories, and the 2-functors, natural transformations, and modifications between them (which form a tricategory) is somewhat scattered, and definitions are seldom given in full explicit detail. One source relevant to the application to TQFT is Chris Schommer-Pries' Ph.D. thesis [43], which collects together all the basic definitions in one place, though even this relies on coherence diagrams which must be found in works by Gordon, Power, and Street [18], and by McCrudden [35]. A recent work by Stay [44] gives a detailed definition and proves that a certain bicategory of spans, related to but distinct from the one we use here, is a symmetric monoidal bicategory. It is also worth noting that the definitions in these works can be seen as a special case of the definition of a tricategory, whose algebraic form is given by Gurski [22].

Even to fully state these definitions is quite lengthy. In particular, to fully define a symmetric monoidal bicategory requires a total of 13 pieces of data, including 1-morphisms such as associators, unitors, and 2-morphisms such as that which takes the place of the pentagon identity for associators in a monoidal category. These various cells, in turn, must satisfy seven coherence conditions expressed by the commutation of various sizeable diagrams of 2 -cells. This does not even include the data implicit in 
the definition of a bicategory without any defined monoidal structure. The definition of a symmetric monoidal 2-functor likewise involves seven pieces of data and five coherence diagrams, beyond those defining a 2-functor (often called "homomorphism") between bicategories.

In what follows, we will not give all this data, nor verify all these conditions explicitly. The reason is that the monoidal structure for our categories is more strict than the completely general case. In many cases, the data in our particular constructions are either just identities, or else are derived from universal properties. In these cases, the coherence properties of such isomorphisms are automatically satisfied because of such universal properties. Rather, the crucial issue will be verifying that we have bicategories and 2-functors at all, and this will occupy most of our attention.

\subsection{Cocycle twisting as homotopy QFT}

The above remarks imply that the action functional associated to $\omega$ will be part of the classical theory, and $\Lambda^{U(1)}$ will be the same 2-functor for all choices of $\omega$. As we shall see, the most natural way to do this uses a generalization of $\operatorname{Span}(\mathbf{G p d})$, which we will call $\operatorname{Span}(\mathbf{G p d})^{U(1)}$, in which groupoids may come equipped with some $U(1)$ cocycle information, which $\Lambda^{U(1)}$ will respect. This contains an isomorphic copy of $\operatorname{Span}(\mathbf{G p d})$ as the sub-bicategory where this extra data is trivial - that is, all cocycles take the constant value $1 \in U(1)$ - on which $\Lambda^{U(1)}$ restricts to $\Lambda$.

This cohomological aspect of the construction of TQFT from Lie groups has been developed in detail by Freed, Hopkins, Lurie and Teleman [16]. We remark that this framework focuses especially on classifying TQFTs in dimension $n$ by means of an element of the $n$th group cohomology of $G$, so since $\Lambda$ and $\Lambda^{U(1)}$ are specifically 2-categorical our construction gives a version of this theory which extends only to codimension-2. The reconstruction of the DW model when $n=3$ is of special interest since 1D manifolds are sufficiently simple to be an interesting stopping point.

Recall that a group cohomology element is an element of the ordinary cohomology of the classifying space:

$$
[\omega] \in H^{3}(B G, U(1))
$$

Its role is best understood in terms of the fact that $G$-connections on a manifold $M$ correspond to homotopy classes of maps $A: M \rightarrow B G$ into the classifying space of $G$. This applies to both manifolds and cobordisms, so we may understand the role of $[\omega]$ in the context of the Homotopy Quantum Field Theory of Turaev [48].

An HQFT is rather like a TQFT, except that the source category consists of manifolds and cobordisms equipped with maps into a target space $X$. Specifically, one has a category $\mathbf{n C o b} / X$, whose objects are $(n-1)$-manifolds equipped with maps into target space $X$. The whose morphisms are cobordisms equipped with maps to $X$ whose restriction to the boundary agrees with the maps from the source and target objects. Then an HQFT in Turaev's sense is a functor from $\mathbf{n C o b} / X$ to Vect.

In the case where $X=B G$, this amounts to saying that an HQFT is an assignment of vector spaces and linear maps to manifolds and cobordisms equipped with a $G$-bundle 
with connection. Such HQFTs are classified by cohomology classes on $X$ ( $B G$ in this case). An equivalent way to say this is that they are classified by gerbes on $X$ (since the cohomology class determines the gerbe).

In a somewhat more specialized case, this has been described by Picken [41], as a "rank-1 TQFT". Picken's construction is given in terms of gluing rules for manifolds with boundary, but is equivalent to the categorical description in terms of composition of cobordisms. Moreover, these are cobordisms are labelled with specific collections of neighborhoods, so that the abstract cohomology class $[\omega]$ (of the map into $B G$ ) is represented by concrete transition functions for a gerbe, relative to these neighborhoods. Being rank-1 means that these transition functions are valued in $U(1)$ - that is, that $\omega$ is a $U(1)$-valued cocycle.

Picken ([41], Theorem 4.6) proves that there is a 1-1 correspondence between such $U(1)$ gerbes, and a certain class of rank-1, 2-dimensional TQFT's. In particular, the formulation given there is helpful in understanding the composition in the new bicategory $\operatorname{Span}(\mathbf{G p d})^{U(1)}$ introduced in Sect. 5.2, since the assignments made using $\omega$ will satisfy certain gluing rules which amount to the characterization of these TQFT's as functors.

In the untwisted situation, the classical field theory $\mathcal{A}_{0}(-)_{G}$ assigns a groupoid $\mathcal{A}_{0}(S)_{G}$ of all connections, which are given by homotopy classes of maps into $B G$ (connections) for each manifold $S$. The exact correspondence is that the mapping space $\operatorname{Maps}(S, B G)$ is the classifying space for this groupoid. The classical field theory when there is a cocycle $\omega$, which we call $\mathcal{A}_{0}(-)_{G}^{\omega}$, will produce the same groupoid. However, it also associates cocycle information to that groupoid, which is derived from $\omega$, as described below. Then, where $\Lambda$ simply performs a sum (or direct sum) over all the objects of this groupoid in a span, the twisted form $\Lambda^{U(1)}$ adjusts these sums using this cocycle information.

Now we describe the category of groupoids with cocycle which we need to make this work.

\subsection{The symmetric monoidal bicategory $\operatorname{Span}(\mathbf{G p d})^{U(1)}$}

In a previous work [37], the author described a monoidal category of groupoids with phases valued in $U(1)$, a special case of groupoids with labels valued in a monoid $M$. The motivation there was to allow for a more physically realistic model of the quantum harmonic oscillator in a category of groupoids and spans. The $U(1)$-phases were needed to get interesting time evolution operators. This involved spans of groupoids equipped with phases derived from a "number-operator", which plays the role of a Hamiltonian for that system. Now we want to describe a variation on this, thinking of phases in the Lagrangian sense, as (exponentiated) actions rather than energies, but an analogous structure is required.

We wish to extend our factorization $\Lambda \circ \mathcal{A}_{0}(-)_{G}=Z_{G}(-)$ to include the twisted case, where a nontrivial Lagrangian is present. So there should be a factorization through a bicategory which contains $\operatorname{Span}(\mathbf{G p d})$. Our bicategory $\operatorname{Span}(\mathbf{G p d})^{U(1)}$ will look like Span $(\mathbf{G p d})$, except that groupoids come equipped with some extra data. 
The simplest such data, most obviously related to Lagrangians in the standard physical sense, is the assignment of an (exponentiated) action in $U(1)$ to a history for a system. That is, the $U(1)$-element is assigned to an object of the groupoid in the middle of a 2-morphism. This function ought to be an invariant of isomorphism classes of objects (physically indistinguishable histories get the same action). When we apply $\Lambda$ to a 2-morphism, we sum over such "histories". In [37] it is explained how groupoidification can be extended to replace groupoid cardinality with a weighted sum:

$$
|(\mathcal{G}, f)|=\sum_{[x] \in \underline{\mathcal{G}}} \frac{f(x)}{|\operatorname{Aut}(x)|}
$$

This is naturally found in $\mathbb{R}^{+} \otimes U(1)$, which we map into $\mathbb{C}$ (identifying all elements $(0, \phi)$ with $0 \in \mathbb{C})$.

This allowed the construction of a full complex Hilbert space in [37]. Now, the role of cardinality in groupoidification arises from the Nakayama isomorphism between the left and right adjoints of the restriction functors. In $\operatorname{Hom}(\mathbf{1}, \mathbf{1})$, as discussed in [39], this isomorphism simply becomes a numerical factor, the groupoid cardinality. Thus, as might be expected, our $\Lambda^{U(1)}$ will incorporate the $U(1)$-valued topological action into a twisting of the Nakayama isomorphism, at the 2-morphism level.

In building $\operatorname{Span}(\mathbf{G p d})^{U(1)}$ as a monoidal bicategory, it is not sufficient simply to take spans in $U(1)$ - Gpd: that is, groupoids with $U(1)$-functions on them. Instead, we need a different structure to describe the appropriate "categorification of the action functional", which will reproduce the twisted DW model. The key point is that $U(1)$ phases on objects can be understood as 0-cocycles in groupoid cohomology.

With our overall aim in mind, we will define a bicategory in which this classical process takes values:

Definition 9 The symmetric monoidal bicategory $\operatorname{Span}(\mathbf{G p d})^{U(1)}$ has:

- Objects: (essentially finite) Groupoids $A$ equipped with 2-cocycles $\theta \in Z^{2}(A, U$ (1)

- 1-Morphisms: A morphism from $\left(A, \theta_{A}\right)$ to $\left(B, \theta_{B}\right)$ is a span of groupoids $A \stackrel{s}{\leftarrow}$ $X \stackrel{t}{\rightarrow} B$, equipped with a 1-cocycle $\alpha \in Z^{1}(X, U(1))$

- 2-morphisms: A 2-morphism from $(X, \alpha, s, t)$ to $\left(X^{\prime}, \alpha^{\prime}, s^{\prime}, t^{\prime}\right)$ in $\operatorname{Hom}\left(\left(A, \theta_{A}\right)\right.$, $\left.\left(B, \theta_{B}\right)\right)$ is an equivalence class of spans of span maps $X \leftarrow Y \rightarrow X^{\prime}$ equipped with 0 -cocycle $\beta \in Z^{0}(Y, U(1))$ (the equivalence is taken up to a $\beta$-preserving groupoid isomorphism, $\left(Y, \beta_{Y}\right) \rightarrow\left(Y^{\prime}, \beta_{Y^{\prime}}\right)$, which commutes with the source and target maps).

This data must satisfy the following conditions:

- In any 1-morphism

$$
(X, \alpha, s, t):\left(A, \theta_{A}\right) \rightarrow\left(B, \theta_{B}\right)
$$

the cocycles satisfy

$$
s^{*} \theta_{A}=t^{*} \theta_{B}
$$


In particular, $\left[s^{*} \theta_{A}\right]=\left[t^{*} \theta_{B}\right]$

- In any 2-morphism

$$
(Y, \beta, \sigma, \tau):\left(X_{1}, \alpha_{1}, s_{1}, t_{1}\right) \Rightarrow\left(X_{2}, \alpha_{2}, s_{2}, t_{2}\right)
$$

the cocycles satisfy

$$
\left(\sigma^{*} \alpha_{1}\right)\left(\tau^{*} \alpha_{2}\right)^{-1}=1
$$

(In particular, $\left[\sigma^{*} \alpha_{1}\right]=\left[\tau^{*} \alpha_{2}\right]$, but moreover, since a 0 -cocycle on a groupoid is an invariant function, $\delta \beta=1 \in U(1)$ and the cocycles themselves are equal.)

The structures making $\operatorname{Span}(\mathbf{G p d})^{U(1)}$ a monoidal bicategory are:

- Composition of 1-morphisms

$$
\left(X_{1}, \alpha_{1}, s_{1}, t_{1}\right):\left(A, \theta_{A}\right) \rightarrow\left(B, \theta_{B}\right)
$$

and

$$
\left(X_{2}, \alpha_{2}, s_{2}, t_{2}\right):\left(B, \theta_{B}\right) \rightarrow\left(C, \theta_{C}\right)
$$

at the object $\left(B, \theta_{B}\right)$ gives the same span of groupoids as in $\operatorname{Span}(\mathbf{G p d})$, and assigns the pullback object the cocycle

$$
\alpha_{1} \cdot \alpha_{2} \cdot \theta_{B}
$$

(explained below)

- Vertical composition of 2-morphisms:

$$
(Y, \beta, \sigma, \tau):\left(X_{1}, \alpha_{1}, s_{1}, t_{1}\right) \rightarrow\left(X_{2}, \alpha_{2}, s_{2}, t_{2}\right)
$$

and

$$
\left(Y^{\prime}, \beta^{\prime}, \sigma^{\prime}, \tau^{\prime}\right):\left(X_{2}, \alpha_{2}, s_{2}, t_{2}\right) \rightarrow\left(X_{3}, \alpha_{3}, s_{3}, t_{3}\right)
$$

at $\left(X_{2}, \alpha_{2}\right)$ gives the same groupoids as in $\operatorname{Span}(\mathbf{G p d})$, with the cocycle given by

$$
\beta \cdot \beta^{\prime} \cdot \alpha_{2}
$$

- Horizontal composition of 2-morphisms:

$$
(Y, \beta, \sigma, \tau):\left(X_{1}, \alpha_{1}, s_{1}, t_{1}\right) \rightarrow\left(X_{2}, \alpha_{2}, s_{2}, t_{2}\right)
$$

in $\operatorname{Hom}\left(\left(A, \theta_{A}\right),\left(B, \theta_{B}\right)\right)$, and 


$$
\left(Y^{\prime}, \beta^{\prime}, \sigma^{\prime}, \tau^{\prime}\right):\left(X_{1}^{\prime}, \alpha_{1}^{\prime}, s_{1}^{\prime}, t_{1}^{\prime}\right) \rightarrow\left(X_{2}^{\prime}, \alpha_{2}^{\prime}, s_{2}^{\prime}, t_{2}^{\prime}\right)
$$

in $\operatorname{Hom}\left(\left(B, \theta_{B}\right),\left(C, \theta_{C}\right)\right)$ at $\left(B, \theta_{B}\right)$ gives the same groupoids as in $\operatorname{Span}(\mathbf{G p d})$, with the cocycle given by

$$
\beta \cdot \beta^{\prime} \cdot \theta_{B}
$$

- The monoidal structure is given by

$$
\left(A, \theta_{A}\right) \otimes\left(B, \theta_{B}\right)=\left(A \times B, \theta_{A} \cdot \theta_{B}\right)
$$

on objects, and on morphisms and 2-morphsims in the same way at each position in the span or span-of-spans diagram.

We explicitly define the cocycle $\alpha_{1} \cdot \alpha_{2} \cdot \theta_{B}$ in (89) as follows. First recall that spans of groupoids are composed by taking the weak pullback of $t_{1}$ and $s_{2}$, which is the isocomma groupoid $t_{1} \downarrow s_{2}$. Its objects are triples $\left(x_{1}, f, x_{2}\right)$ where $f: t_{1}\left(x_{1}\right) \rightarrow s_{2}\left(x_{2}\right) \in$ $B$, and its morphisms are pairs $\left(g_{1}, g_{2}\right) \in X_{1} \times X_{2}$, forming commuting squares in $B$ :

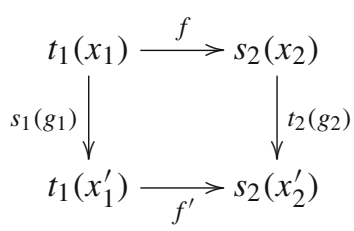

For 1-morphisms $(X, \alpha, s, t):\left(A, \theta_{A}\right) \rightarrow\left(B, \theta_{B}\right)$, the defining property of the 1-cocycle $\alpha$ is that it determines a functor $\alpha: X \rightarrow U(1)$, where $U(1)$ is understood as a groupoid with one object. On the other hand, the 2-cocycle $\theta_{B}$ is a map from pairs of morphisms $\left(f, f^{\prime}\right) \in B$ to $U(1)$ satisfying the 2-cocycle property, which ensures that "twisting" multiplication by $\theta_{B}$ remains associative if it was so originally.

Then the functor $\alpha_{1} \cdot \alpha_{2} \cdot \theta_{B}:\left(t_{1} \downarrow s_{2}\right) \rightarrow U(1)$ assigns the 1-morphism (97) the value:

$$
\alpha_{1}\left(g_{1}\right) \cdot \alpha_{2}\left(g_{2}\right) \cdot \theta_{B}\left(f, f^{\prime}\right)
$$

(This is the meaning of "twisting multiplication by $\theta_{B}$ " above).

The horizontal composition rule for 2-morphisms is similar, in that we compose by weak pullback over $\left(B, \theta_{B}\right)$ for both 1-morphisms and 2-morphisms. All the twistings by $\theta_{B}$ are compatible under the maps of the 2-morphism's inner span.

The vertical composition rule for 2-morphisms is also similar, except that the 0cocycle is assigned to objects of $Y^{\prime} \circ Y$. These are again of the form $\left(y^{\prime}, f, y\right)$ where $f: \sigma^{\prime}\left(y^{\prime}\right) \rightarrow \tau(y) \in X_{2}$. So the composite cocycle assigns this object the value $\beta^{\prime}\left(y^{\prime}\right) \cdot \alpha_{2}(f) \cdot \beta(y)$.

This composition rule may be surprising at first sight, in that one might naively expect the cocycle on $Y^{\prime} \circ Y$ to be $\beta^{\prime} \cdot \beta$, or on $X_{2} \circ X_{1}$ to be $\alpha_{2} \cdot \alpha_{1}$, so that a 
0 -cocycle is a product only of 0 -cocycles, and so on. It is clear, though, that objects in the $\tau \downarrow \sigma^{\prime}$ contain a morphism from $X_{2}$, or that $t_{1} \downarrow s_{2}$ contains two morphisms from $B$, so this rule is a consequence of the use of the weak pullback to compose spans of groupoids. We will describe this in terms of transition functions in the case of groupoids of connections in Sect. 5.3. For the moment, the intuitive idea is that in the composite of two spans of groupoids, objects in the $Y$ or the $X_{i}$ need not match exactly as in a fibered product, and some "twisting" is needed to align them correctly. As we will see, this is necessary to ensure that the composition rules indeed determine cocycles on the groupoids found by such weak pullback.

Of course, we must check that this structure indeed defines a symmetric monoidal bicategory. To see this, it is useful to recall why $\operatorname{Span}(\mathbf{G p d})$ is one.

\section{Lemma $2 \operatorname{Span}(\mathbf{G p d})$ is a symmetric monoidal bicategory.}

Proof The main principle used is the fact that composition of spans is given by weak pullback, is defined by a universal property. The unique canonical maps from this property gives provides associator and unitor 2-cells for composition, which we do not name explicitly. They arise from the canonical maps from a weak pullback, expressed as spans with one identity leg. The fact that they are determined by a universal property ensures that they satisfy the necessary coherence conditions. For example, the pentagon identity asserts that two composites are equal, which in this case are both the same canonical map from the universal property of the pullback.

The monoidal structure on $\operatorname{Span}(\mathbf{G p d})$, denoted by $\otimes$, is given on objects by the Cartesian product in Gpd (which is not a categorical product in $\operatorname{Span}(\mathbf{G p d})$ ). This extends naturally to spans. Again, a monoidal bicategory must have associator and unitor cells for the monoidal product. We take these to be precisely the canonical maps which come from the universal property of the product in Gpd, interpreted as morphisms in $\operatorname{Span}(\mathbf{G p d})$ with one leg the identity map. For this reason, we do not explicitly name them.

The definition of a monoidal bicategory then demands the existence of various 2-cells which take the place of the pentagon identity, unitor identites, and other equations which hold in monoidal categories for composites of associators and unitors. There is a slight complication in defining these, since the composite of 1-morphisms, by weak pullback, is only defined up to an isomorphism 2-cell.

However, since the associator and unitor 1-morphisms come from maps in Gpd, we may adopt the convention to choose the composite of the spans which comes directly from the composite of the corresponding maps. In this case, the pentagon and other coherence 2-cells in Span (Gpd) are just the identities, since they compare two composites which give canonical isomorphisms that come from the universal property of the product. These coherence 2-cells will therefore automatically satisfy the axioms for a symmetric monoidal bicategory, which each relate two identity diagrams. (Different choices of composites will give 2-cells which differ from these by the isomorphisms to our canonical choice of composite.)

The symmetry data for $\operatorname{Span}(\mathbf{G p d})$ is trivial in a similar way. The braiding 1morphism $\beta: A \otimes B \rightarrow B \otimes A$ is just the span which comes from the canonical morphism in Gpd which comes from the universal property of the product. 
A symmetric monoidal bicategory has two hexagonal 2-cells relating composites of $\beta$ and the associator (taking the role of the hexagon identity for symmetric monoidal categories). Again, this structure is strict and we can choose the identity maps, provided we make the canonical choice of composites. For any other choice of weak pullback, these 2-isomorphisms are the canonical isomorphism to that canonical choice. Again, identities will automatically satisfy the coherence condition, and thus so will these other choices in case we make non-canonical choices for composites of 1-morphisms.

Finally, the braiding map is strictly symmetric, so as above, given canonical choices for the corresponding spans, the structural 2-isomorphism $\sigma: I \rightarrow \beta \circ \beta$ is just the identity.

Structures in $\operatorname{Span}(\mathbf{G p d})^{U(1)}$ are exactly those in $\operatorname{Span}(\mathbf{G p d})$, together with cocycle data. The previous lemma shows that all the properties for a symmetric monoidal bicategory hold when the cocycle data is trivial. It remains to check that the properties still hold when nontrivial cocycle data is added.

Lemma $3 \operatorname{Span}(\mathbf{G p d})^{U(1)}$ is a symmetric monoidal bicategory.

Proof We note that the underlying spans and spans-of-spans which define the data of the symmetric monoidal bicategory $\operatorname{Span}(\mathbf{G p d})^{U(1)}$ will be exactly those which appear in $\operatorname{Span}(\mathbf{G p d})$. We need only define the associated cocycle data and see that the axioms still hold.

Now, $\operatorname{Span}(\mathbf{G p d})^{U(1)}$ is closed under composition of 1-morphisms if (89) determines a 1-cocycle, namely $\alpha_{1} \cdot \alpha_{2} \cdot \theta_{B}$ is a functor from $\left(t_{1} \downarrow s_{2}\right)$ into $U(1)$. Since $\alpha_{1}$ and $\alpha_{2}$ are functors, this follows precisely from the fact that $\theta_{B}$ is a 2-cocycle, so that the twisted multiplication remains associative. In the same way, (92) determines an invariant function on objects of ( $\tau \downarrow \sigma^{\prime}$ ) (that is, a 0 -cocycle), since $\alpha_{2}$ is a 1-cocycle, so its coboundary is trivial.

Now, the structure morphisms (associator and unitors) for 1-morphism composition in $\operatorname{Span}(\mathbf{G p d}) U(1)$ is just the same as those for $\operatorname{Span}(\mathbf{G p d})$, with the trivial cocycle (i.e. constant of value 1). This is because the cocycle given by the two composition orders is preserved by the canonical isomorphism between the composites, so the identity (86) holds with the trivial cocycle. (A similar argument shows that composition of 2-morphisms is associative and has identities).

Similarly, the fact that (96) defines a monoidal product follows from the fact that the Cartesian product $\times$ on $\mathbf{G p d}$ determines a monoidal product in $\operatorname{Span}(\mathbf{G p d})$, and multiplication is a monoid operation for $U(1)$. The fact that this monoidal product is symmetric follows from the symmetry isomorphism from the universal property of $\times$ and commutativity of multiplication in $U(1)$. The monoidal unit is $(\mathbf{1}, 1)$, as can easily be verified.

An obvious but important fact is:

Corollary 1 The symmetric monoidal bicategory $\operatorname{Span}(\mathbf{G p d})^{U(1)}$ contains Span (Gpd) as a sub-symmetric monoidal bicategory.

Proof There is a fully faithful symmetric monoidal 2-functor embedding any object, morphism, or 2-morphism of $\operatorname{Span}(\mathbf{G p d})$ into $\operatorname{Span}(\mathbf{G p d})^{U(1)}$ taking any groupoid to 
the same groupoid equipped with the trivial cocycle which has the constant value 1 , and leaving all maps in spans unchanged. This is clearly a functor, since all operations in $\operatorname{Span}(\mathbf{G p d})^{U(1)}$ are just the same as those in $\operatorname{Span}(\mathbf{G p d})$ when cocycles are disregarded. The image of this embedding is a sub-category since it contains all identities and the monoidal unit, and is closed under the composition and monoidal operations of $\operatorname{Span}(\mathbf{G p d})^{U(1)}$.

Knowing that $\operatorname{Span}(\mathbf{G p d})^{U(1)}$ is a symmetric monoidal bicategory, we want to construct the two symmetric monoidal 2-functors $\mathcal{A}_{0}(-)_{G}^{\omega}$ [given a fixed 3-cocycle $\left.\omega \in Z^{3}(B G, U(1))\right]$, and $\Lambda^{U(1)}$. We address these next.

\subsection{The classical field theory}

Our construction of the ETQFT $Z_{G}^{\omega}$ corresponding to the twisted DW model will use a generalization of the factorization $Z_{G}(-)=\Lambda \circ \mathcal{A}_{0}(-)_{G}$. The quantization functor $\Lambda^{U(1)}: \operatorname{Span}(\mathbf{G p d})^{U(1)} \rightarrow \mathbf{2 V e c t}$ will always be the same, and the cocycle $\omega$ will modify only the classical field theory, by defining the cocycle data in $\operatorname{Span}(\mathbf{G p d})^{U(1)}$.

We will begin by describing the classical field theory component.

The "topological action" for the twisted DW theory comes from a $U(1)$-valued class in group cohomology:

$$
[\omega] \in H_{g p}^{3}(G, U(1))
$$

which we can take as represented by some particular cocycle:

$$
\omega \in Z_{g p}^{3}(G, U(1))
$$

Now, group cohomology is just the usual third cohomology of the classifying space of $\mathrm{G}$, so in fact this says:

$$
\omega \in Z^{3}(B G, U(1))
$$

This is a function which, given a 3-cycle in the space $B G$, defines a number in $U(1)$, satisfying the cocycle condition. It is usual to think of the classifying space defined simplicially, and therefore to consider what $\omega$ does to 3-simplices.

Now, the classical part of the DW construction with associated 3-cocycle $\omega$ gives cocycles of different degree associated to the groupoids of connections for manifolds of different dimension. In general, a $k$-dimensional cobordism will produce a groupoid (as object, or part of a span) which has a $(3-k)$-cocycle associated to it. This data arises from the "transgression" of the original cocycle, an algebraic structure explained very nicely by Willerton [49]. We briefly summarize it here.

First, this concept uses the fact that a (flat) connection on a $k$-dimensional manifold $M$ can be understood as a homotopy class of maps into the classifying space, $f$ : $M \rightarrow B G$. Recall that an indirect definition of $B G$ is precisely this fact. The classifying space functor $B$ is right adjoint to the fundamental-groupoid functor $\Pi_{1}$, so that in 
particular $\operatorname{Hom}\left(\Pi_{1}(M), G\right) \cong \operatorname{Hom}(M, B G)$, where the second term consists of homotopy classes of maps of spaces. The most important feature of $B G$ is that its fundamental group is $G$ and all other homotopy groups are trivial. More concrete constructions of $B G$ for particular $G$ depend on exactly which category of spaces $B G$ is considered to lie in.

One standard choice, used in the "bar construction" is that $B G$ is a simplicial set. It is constructed by taking a single base-point (if $G$ is a group, or one base-point for each object if $G$ is a groupoid), adding edges for each element of $G$, and then adjoining higher-dimensional cells as necessary to make sure there are no higher homotopy groups. For instance, one would add: a triangular face adjoining edges $f, g$ and $f g$ to make this loop contractible; a tetrahedron between four such triangles expressing each associativity relation; and so on. For convenience, we take $B G$ as presented as such a simplicial complex. A 3-cocycle $\omega$ on $B G$ then gives a value in $U$ (1) to each 3-simplex $\Delta_{3}$ in $B G$. (A smooth realization of $B G$ will treat this as an integral of some 3-form over the 3-chain $\Delta_{3}$. In general, when describing integration in the group $U(1)$, we will treat it as the additive group $\mathbb{R} / \mathbb{Z}$ ).

Now, a connection is given by a point in the space $\operatorname{Maps}(M, B G)$. This space in turn is a simplicial complex, and in fact is the classifying space of the groupoid of flat connections on $M$ (each isomorphism class of objects corresponds to a connected component of this space given by one of the base-points). Since a point in $\operatorname{Maps}(M, B G)$ is a function, there is the evaluation map:

$$
\text { ev }: M \times \operatorname{Maps}(M, B G) \rightarrow B G
$$

The image of $f: M \rightarrow B G$, or rather of $M \times f$, under $e v$ is then a $k$-chain (perhaps degenerate) in $B G$, namely the image $f(M)$. If we take a $(3-k)$-simplex $\Delta_{3-k}$ in $\operatorname{Maps}(M, B G)$, then these images in $B G$ form a 3-dimensional subspace which looks like $M \times \Delta_{3-k}$. This can be decomposed into individual simplices in $B G$.

But then, this means we have a $(3-k)$-cocycle on $\operatorname{Maps}(M, B G)$, the "transgression" of $\omega$ to $\operatorname{Maps}(M, B G)$, which is denoted:

$$
\tau_{M}(\omega) \in H^{3-k}(\operatorname{Maps}(M, B G), U(1))
$$

It is given by integrating $\omega$ :

$$
\tau_{M}(\omega)=\int_{M} e v^{*}(\omega)
$$

It can be integrated over $\Delta_{3-k}$ to get an element of $U(1)$. Our classical field theory will assign the cocycle $\tau_{M}(\omega)$ to each groupoid $\mathcal{A}_{0}(M)_{G}$.

So for 2-morphisms in $\mathbf{3} \mathbf{C o b}_{\mathbf{2}}$, which are 3-dimensional cobordisms $M$ ("spacetimes with boundary"), this just amounts to an action functional: a $U$ (1)-valued function on connections. In the untwisted case, we have the constant function $\omega \cong 1$, and thus $\tau_{M}(\omega) \cong 1$ also. But for objects (1-dimensional manifolds) and morphisms (2-dimensional cobordisms), we get different data: respectively, 2-cocycles and 1-cocycles. 
Definition 10 For a fixed finite group $G$ and group 3-cocycle $\omega$, the classical field theory is a symmetric monoidal 2-functor:

$$
\mathcal{A}_{0}(-)_{G}^{\omega}: \mathbf{3 C o b}_{2} \rightarrow \operatorname{Span}(\mathbf{G p d})^{U(1)}
$$

which acts as follows:

- Objects: $\mathcal{A}_{0}(B)_{G}^{\omega}=\left(\mathcal{A}_{0}(B)_{G}, \tau_{B}(\omega)\right)$

- Morphisms: $\mathcal{A}_{0}\left(S: B_{1} \rightarrow B_{2}\right)_{G}^{\omega}=\left(\mathcal{A}_{0}(S)_{G}, \tau_{S}(\omega), i_{1}^{*}, i_{2}^{*}\right)$ (where the $i_{j}$ are the inclusion maps of the $B_{j}$ into $S$ ).

- 2-Morphisms: $\mathcal{A}_{0}\left(M: S \rightarrow S^{\prime}\right)_{G}^{\omega}=\left(\mathcal{A}_{0}(M)_{G}, \tau_{M}(\omega), i^{*},\left(i^{\prime}\right)^{*}\right)$, where again $i$ and $i^{\prime}$ are inclusion maps of $S$ and $S^{\prime}$ into $M$.

This definition implicitly makes the assertion that this is a symmetric monoidal 2-functor. The first thing to check is that it exists at all.

Lemma 4 The construction for $\mathcal{A}_{0}(-)_{G}^{\omega}$ gives well-defined maps for objects, morphisms, and 2-morphisms into $\operatorname{Span}(\mathbf{G p d})^{U(1)}$.

Proof We need to check that the image of $\mathcal{A}_{0}(-)_{G}^{\omega}$ actually lies in $\operatorname{Span}(\mathbf{G p d})^{U(1)}$. It is well-known that transgression will yield cocycles (see e.g. [49]), so we need to verify the conditions (84) and (86) for those cocycles.

Suppose that $S: B \rightarrow B^{\prime}$ is a cobordism, so that $\partial S=B \sqcup B^{\prime}$, and applying $\mathcal{A}_{0}(-)_{G}^{\omega}$ we get the span:

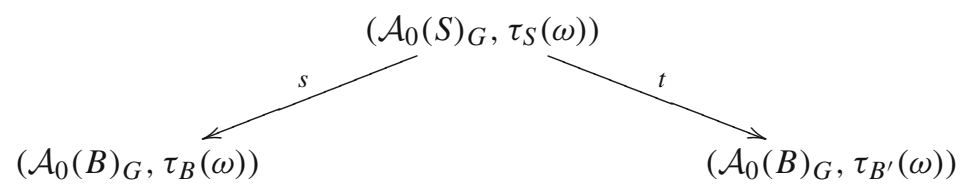

Then we want to verify that the cocycles are compatible, or in other words that $\left(s^{*} \theta_{B}\right)\left(t^{*} \theta_{B^{\prime}}\right)^{-1}=1$. Restating this with the cocycle taking values in the additive group $\mathbb{R} / \mathbb{Z}$ (since we want to express the value in terms of an integral):

$$
s^{*} \theta_{B}-t^{*} \theta_{B^{\prime}}=0
$$

But this is a 2-cocycle on $\mathcal{A}_{0}(S)_{G}$ given by:

$$
\begin{aligned}
& \left(s^{*} \theta_{B}\right)-\left(t^{*} \theta_{B^{\prime}}\right) \\
& =s^{*}\left(\int_{B} e v^{*}(\omega)\right)-t^{*}\left(\int_{B^{\prime}} e v^{*}(\omega)\right) \\
& =\pi^{*}\left(\int_{\partial S} e v^{*}(\omega)\right)
\end{aligned}
$$


Here, we are using the fact that the orientation on $B$ and $B^{\prime}$, the boundary components of $S$, are opposite, by convention and denoting by $\pi=s \otimes \bar{t}$ the projection map from $\mathcal{A}_{0}(S)_{G}$ to $\mathcal{A}_{0}(\partial S)_{G}$. So this says the difference of $s^{*} \theta_{B}$ and $t^{*} \theta_{B^{\prime}}$ is $\pi^{*}\left(\tau_{\partial S}(\omega)\right)$.

This is the pullback of a 2-cocycle on $\operatorname{Maps}(\partial S, B G)$ to a 2-cocycle on $\operatorname{Maps}(S, B G)$. Now suppose we evaluate it on a 2-chain $\Delta_{2}$ in $\operatorname{Maps}(S, B G)$, which we take to be a 2-simplex (a similar proof would work for non-simplicial constructions of $B G)$. Then:

$$
\begin{aligned}
\pi^{*} & \left(\tau_{\partial S}(\omega)\right)\left[\Delta_{2}\right] \\
= & \int_{\partial S \times \Delta_{2}} e v^{*}(\omega) \\
= & \int_{e v\left(\partial S \times \Delta_{2}\right)}(\omega)
\end{aligned}
$$

This is an integral of $\omega$ on a 3-chain in $B G$ which is one part of:

$$
\partial\left(S \times \Delta_{2}\right)=\left(\partial S \times \Delta_{2}\right) \cup\left(S \times \partial \Delta_{2}\right)
$$

So if we evaluate on the whole 3-chain, we have:

$$
\begin{aligned}
& \int_{e v\left(\partial\left(S \times \Delta_{2}\right)\right)}(\omega) \\
= & \int_{e v\left(\left(\partial S \times \Delta_{2}\right) \cup\left(S \times \partial \Delta_{2}\right)\right)}(\omega) \\
= & \int_{e v\left(\left(\partial S \times \Delta_{2}\right)\right.}(\omega)+\int_{e v\left(\left(S \times \partial \Delta_{2}\right)\right)}(\omega)
\end{aligned}
$$

Now, since

$$
\int_{e v\left(S \times \partial \Delta_{2}\right)}(\omega)=\tau_{S}(\omega)\left[\delta \Delta_{2}\right]
$$

which is the evaluation of the 1-cocycle $\tau_{S}(\omega)$ on a boundary, this part is equal to 0 . However, by Stokes' theorem:

$$
\int_{e v\left(\partial\left(S \times \Delta_{2}\right)\right)}(\omega)=\int_{e v\left(S \times \Delta_{2}\right)} \delta(\omega)
$$

and since $\omega$ is a cocycle, this is again 0 . Thus, we have

$$
\pi^{*}\left(\tau_{\partial S}(\omega)\right)\left[\Delta_{2}\right]=0
$$


so that finally $s^{*} \theta_{B}=t^{\prime *} \theta_{B^{\prime}}$ as required.

A similar argument with 1-cocycles and 0-cocycles holds at the level of 2-morphisms.

Now we have that $\mathcal{A}_{0}(-)_{G}^{\omega}$ gives well-defined maps of objects, morphisms, and 2-morphisms from $\mathbf{3 C o b _ { 2 }}$ into $\operatorname{Span}(\mathbf{G p d})^{U(1)}$.

The next lemma verifies that $\mathcal{A}_{0}(-)_{G}^{\omega}$ is indeed a symmetric monoidal 2-functor, but some preliminary remarks may be useful.

We know that $\mathcal{A}_{0}(-)_{G}: \mathbf{3} \mathbf{C o b}_{2} \rightarrow \operatorname{Span}(\mathbf{G p d})$ is a symmetric monoidal 2-functor, since it comes from taking maps into $B G$. Functoriality follows because this is local, and turns (weak) pushouts into (weak) pullbacks. To verify the analogous fact for $\mathcal{A}_{0}(-)_{G}^{\omega}$, we first need to check that the cocycle data makes it well-defined.

As mentioned in Sect. 5.1 that the cocycle values themselves associated to manifolds and cobordisms with connection, by Picken's construction, define an HQFT. The properties proved in [41] amount to the fact that such an HQFT is a (symmetric) monoidal functor into vector spaces (1-dimensional in this case) from a category of manifolds and cobordisms which are equipped with a map into a target space $X$, which in this case is $B G$. Such HQFT are in 1-1 correspondence with gerbes on $B G$, which are determined by cocycles such as $\omega$, which represent the curvature form for the gerbe.

Given $\mathcal{A}_{0}(-)_{G}^{\omega}$, the composition rule for $\operatorname{Span}(\mathbf{G p d})^{U(1)}$ discussed in the previous section gets a useful geometric interpretation. In the special case where 1-morphisms are manifolds without boundary, seen as cobordisms from the empty manifold to itself, the cocycle data for any groupoid of connections may be seen as a by a rank-1 embedded 2-dimensional TQFT with target $B G$ in the sense of Picken (definition 4.1 of [41]). This may be understood as a (unitary) TQFT (or rather, HQFT, since manifolds and cobordisms are equipped with maps to $B G$ ) in which every vector space is just the 1-dimensional vector space $\mathbb{C}$. (Note that the adjective "unitary" is unnecessary for finite groups, since every element has finite order so all values are in fact roots of unity.)

Thus, one gets an element of $U(1)$ for each morphism of objects (i.e. between 1-manifolds equipped with connection - in other words, for a gauge transformation), by what Picken calls $Z^{\prime}$ (part of $Z$ in our terminology), and an element of $U$ (1) for each cobordism by Picken's $Z$. Then the composition rule for cobordisms is Picken's gluing rule, which agrees with our composition rule (92) in that case. The point here is that one gets an extra contribution from the boundary $B$ where two manifolds are being glued. This is explained in [41] in terms of transition functions (for a gerbe induced from the gerbe on $B G$ classified by $\omega$ ). Essentially, one must make a gauge transformation to ensure that connections on the two cobordisms being glued actually match at the boundary. We may also understand it by thinking of the gauge transformation identifying the different connections on $B$ as a mapping cylinder: two copies of $B$ with connections in different gauge, are identified with the ends of a cylinder $B \times I$. This has a nontrivial connection where the holonomies along the edge $b \times I$ for each point $b \in B$ conjugates an holonomy for a loop based at that point.

This is the extra morphism in the weak pullback contributing to this composition, as described in Sect. 5.2. 
This is the idea behind the proof of the following.

Theorem 3 The construction $\mathcal{A}_{0}(-)_{G}^{\omega}$ gives a symmetric monoidal 2-functor.

Proof First, we check that composition of 1-morphisms is preserved up to isomorphism. Suppose $S: B_{1} \rightarrow B_{2}$ and $S^{\prime}: B_{2} \rightarrow B_{3}$ are cobordisms. Then

$$
\mathcal{A}_{0}\left(S^{\prime} \circ S\right)_{G}^{\omega}=\left(\mathcal{A}_{0}\left(S^{\prime} \circ S\right)_{G}, \tau_{S^{\prime} \circ S}(\omega), i_{1}^{*}, i_{3}^{*}\right)
$$

On the other hand, since we have $\mathcal{A}_{0}\left(B_{2}\right)_{G}^{\omega}=\left(\mathcal{A}_{0}(B)_{G}, \theta_{B_{2}}\right)$ and $\theta_{B_{2}}=\tau_{B_{2}}(\omega)$, it follows from (89) that:

$$
\begin{aligned}
& \mathcal{A}_{0}\left(S^{\prime}\right)_{G}^{\omega} \circ \mathcal{A}_{0}(S)_{G}^{\omega} \\
& \left.=\left(\mathcal{A}_{0}\left(S^{\prime}\right)_{G}, \tau_{S^{\prime}}(\omega), i_{1}^{*}, i_{2}^{*}\right)\right) \circ\left(\mathcal{A}_{0}(S)_{G}, \cdot \tau_{S}(\omega), i_{2}^{*}, i_{3}^{*}\right) \\
& \cong\left(\mathcal{A}_{0}\left(S^{\prime} \circ S\right)_{G}, \tau_{S^{\prime}}(\omega) \cdot \tau_{S}(\omega) \cdot \tau_{B_{2}}(\omega), I_{1}^{*}, I_{3}^{*}\right)
\end{aligned}
$$

The composition of spans is just the weak pullback over $\mathcal{A}_{0}\left(B_{2}\right)_{G}$. The $I_{j}$ are the inclusion maps of boundaries into the composite cobordism.

Since we know $\mathcal{A}_{0}\left(S^{\prime} \circ S\right)_{G} \cong \mathcal{A}_{0}\left(S^{\prime}\right)_{G} \circ \mathcal{A}_{0}(S)_{G}$, it suffices to check that

$$
\tau_{S^{\prime} \circ S}(\omega)=\tau_{S^{\prime}}(\omega) \cdot \tau_{S}(\omega) \cdot \tau_{B_{2}}(\omega)
$$

under this identification. This is a 1-cocycle on the groupoid $\mathcal{A}_{0}\left(S^{\prime} \circ S\right)_{G}$ of connections on the composite. That is, a $U(1)$-valued function on gauge transformations which respects their composition.

Now, we are identifying the groupoid of connections on $S^{\prime} \circ S$ with the weak pullback of $\mathcal{A}_{0}\left(S^{\prime}\right)_{G}$ and $\mathcal{A}_{0}(S)_{G}$ over $\mathcal{A}_{0}\left(B_{2}\right)_{G}$ (to which it is naturally equivalent). This means a connection on the whole space is determined by a pair of connections in $\mathcal{A}_{0}\left(S^{\prime}\right)_{G} \times \mathcal{A}_{0}(S)_{G}$ identified by a gauge transformation between the restrictions of the connections to $B_{2}$. (That is, there is a "transition function" specifying the change of gauge when gluing the connections at $B_{2}$ ). A gauge transformation between two such objects is then a square of the form (97), and includes two gauge transformations from $\mathcal{A}_{0}\left(B_{2}\right)_{G}$ - the transition function for the gauge transformations. As in the discussion of Picken's HQFT above, this is assigned an element of $U(1)$, just as if we glued using a mapping cylinder $B_{2} \times I$ with a nontrivial connection. This factor is precisely $\tau_{B_{2}}(\omega)$ by this construction. The cocycle on $\mathcal{A}_{0}\left(S^{\prime} \circ S\right)_{G}$ is exactly this cocycle (pulled back through the equivalence with $\left.\mathcal{A}_{0}\left(S^{\prime}\right)_{G} \circ \mathcal{A}_{0}(S)_{G}\right)$.

A similar argument for 0-cocycles and gluing along 1-cocycles shows the composition of 2-morphisms is respected. Together these imply the preservation of all identity 1 - and 2-morphisms.

It is straightforward to verify that there is a canonical isomorphism $\mathcal{A}_{0}(A \sqcup B)_{G}^{\omega} \cong$ $\mathcal{A}_{0}(A)_{G}^{\omega} \otimes \mathcal{A}_{0}(B)_{G}^{\omega}$ (often called $H$, as in [43]). This is because the monoidal product in Span(Gpd) is just the Cartesian product from Gpd, and the same canonical isomorphism, seen as a span, will work here. Moreover, in the product, the cocycles simply 
multiply in $U(1)$. On the other hand, the transgressed cocycles are

$$
\begin{aligned}
\tau_{A \sqcup B}(\omega) & =\int_{A \sqcup B} e v^{*}(\omega) \\
& =\int_{A} e v^{*}(\omega)+\int_{B} e v^{*}(\omega) \\
& =\tau_{A}(\omega)+\tau_{B}(\omega)
\end{aligned}
$$

Since this sum is in $\mathbb{R} / \mathbb{Z}$, this is exactly what we expect. Likewise, the monoidal unit is preserved up to a canonical isomorphism.

These structural isomorphisms naturally satisfy all the coherence conditions for a functor of monoidal bicategories (as in [18]) by the universal property, and the fact that the cocycles multiply strictly. A similar argument holds for the invertible modification relating the monoidal structure map $H$ and the symmetry map.

Thus, $\mathcal{A}_{0}(-)_{G}^{\omega}$ is a symmetric monoidal 2-functor.

\subsection{The twisted 2-linearization functor $\Lambda^{U(1)}$}

We have suggested that the twisted classical theory behind the DW model takes values in $\operatorname{Span}(\mathbf{G p d})^{U(1)}$. We now want to understand the twisted analog of $\Lambda$, the 2-linearization, or "quantization" functor $\Lambda^{U(1)}$.

The essential point is that we use the representations of the twisted groupoid algebras such as $\mathbb{C}^{\theta_{A}}[A]$. This is the algebra of complex functions on morphisms of $A$, with the "twisted" multiplication:

$$
\left(F \star_{A} G\right)(f)=\sum_{g} F(g) G\left(g^{-1} f\right) \theta_{A}\left(g, g^{-1} f\right)
$$

The sum is taken over all morphisms $g \in A$ whose target is the source of $f$ : this is a twisted form of the usual convolution product. Given this notation, it is a standard fact that representations of this twisted algebra correspond to so-called "twisted representations" $\rho$ of the groupoid itself, in which the usual composition rule is replaced by $\rho\left(g_{1}\right) \circ \rho\left(g_{2}\right)=\theta_{A}\left(g_{1}, g_{2}\right) \rho\left(g_{1} \circ g_{2}\right)$. It is also possible to describe these as representations of a central extension of the groupoid (more usual in the case of a group). We will choose the first of these descriptions for convenience.

So 2-cocycles on objects twist the representation categories that appear as the output of $\Lambda$. The 1-cocycles, as we will see, twist the functors between them associated to spans, and the 0-cocycles twist the natural transformations.

In particular, 1-morphisms will involve restriction and induction of these twisted representations, for example pulling back along $s:\left(X, \alpha_{X}\right) \rightarrow\left(A, \theta_{A}\right)$ turns a representation of $\mathbb{C}^{\theta_{A}}[A]$ into a representation of $\mathbb{C}^{s^{*} \theta_{A}}[X]$. By preceding arguments, this is the same as $\mathbb{C}^{t^{*} \theta_{B}}[X]$ since $\left.s^{*} \theta_{A}=t^{*} \theta_{B}\right)$.

It is possible to repeat what $\Lambda$ does to a span $(X, s, t): A \rightarrow B$, which simply takes $t_{*} \circ s^{*}$ : pull back a representation to $X$ and push forward to $B$. However, if a cocycle 
$\alpha_{X}$ is present, we can "twist" this identification of $\mathbb{C}^{s^{*} \theta_{A}}[X]$ with $\mathbb{C}^{t^{*} \theta_{B}}[X]$ by $\alpha_{X}$. This uses the map:

$$
M_{\alpha}: \mathbb{C}^{s^{*} \theta_{A}}[X] \rightarrow \mathbb{C}^{t^{*} \theta_{B}}[X]
$$

which takes $f: X \rightarrow \mathbb{C}$ to $\alpha \cdot f: X \rightarrow \mathbb{C}$. Of course, this is actually an automorphism of one algebra, since the twisting cocycles are actually equal by the condition (84). It is convenient, however, to represent $M_{\alpha}$ this way in what follows. A well-known but still crucial fact which we demonstrate here, is:

Proposition $5 M_{\alpha}$ is an algebra isomorphism.

Proof Clearly $M_{\alpha}$ is linear, so we check compatibility with the products. Suppose $F, G: X \rightarrow \mathbb{C}$, are thought of as elements of $\mathbb{C}^{s^{*} \theta_{A}}[X]$, with the product (119). Then applying $M_{\alpha}$, at $f \in X$ we have:

$$
\begin{aligned}
\left(M_{\alpha}(F) \star_{B}\left(M_{\alpha}(G)\right)(f)\right. & =\sum_{g}(\alpha \cdot F)(g)(\alpha \cdot G)\left(g^{-1} f\right) \theta_{B}\left(g, g^{-1} f\right) \\
& =\sum_{g} \alpha(g) F(g) \alpha\left(g^{-1} f\right) G\left(g^{-1} f\right) \theta_{B}\left(g, g^{-1} f\right) \\
& =\sum_{g} F(g) G\left(g^{-1} f\right)(\delta \alpha)\left(g, g^{-1} f\right)^{-1} \cdot \theta_{A}\left(g, g^{-1} f\right) \\
& =\sum_{g} F(g) G\left(g^{-1} f\right) \alpha(f) \cdot \theta_{A}\left(g, g^{-1} f\right) \\
& =\alpha(f) \cdot\left(F \star_{A} G\right)(f) \\
& =\left(M_{\alpha}\left(F \star_{A} G\right)\right)(f)
\end{aligned}
$$

And indeed, since this map is plainly invertible with inverse $M_{\alpha^{-1}}$, this gives an isomorphism between the two algebras.

This isomorphism induces a specific (contravariant) isomorphism between the representation categories, by pre-composition:

$$
M_{\alpha}^{*}: \operatorname{Rep}\left(X, s^{*} \theta_{A}\right) \rightarrow \operatorname{Rep}\left(X, t^{*} \theta_{B}\right)
$$

We will use this in the construction for the 1-morphism map of $\Lambda^{U(1)}$.

Just as 2-cocycles twist the objects (representation categories) and 1-cocycles twist the 1-morphisms (functors), so the 0-cocycles will twist 2-morphisms (natural transformations). The untwisted $\Lambda$ uses the unit and counit for the adjunction between induction and restriction functors of representations along groupoid homomorphisms. There is still an adjunction for algebra representations, so this part is much the same. However, $\Lambda$ also uses the Nakayama isomorphism, as in (16). This is a canonical choice of isomorphism between the left and right adjoints to the restriction functor.

However, since 2Vect is enriched in Vect $\mathbb{C}_{\mathbb{C}}$, we can of course "twist" this natural isomorphism by a scalar that depends on a choice of object. This is exactly the role of 
the 0-cocycle (which is physically interpreted as the complex-valued "action" for the configuration of our QFT that object represents).

Definition 11 The "twisted form" of the Nakayama isomorphism:

$$
N_{\beta_{Y}}: \sigma_{*} \circ\left(M_{\sigma^{*} \alpha_{1}}\right)^{*} \circ \sigma^{*} \Longrightarrow \tau_{*} \circ\left(M_{\tau^{*} \alpha_{2}}\right)^{*} \circ \tau^{*}
$$

which relates the ( $\alpha$-twisted) forms of the left and right adjunction acts at each object $y \in Y$ by:

$$
N_{\beta_{Y}}: \bigoplus_{[y] \mid f(y) \cong x} \phi_{y} \mapsto \bigoplus_{[y] \mid f(y) \cong x} \frac{\beta_{Y}(y)}{\# A u t(y)} \sum_{g \in A u t(x)} g \otimes \phi_{y}\left(g^{-1}\right)
$$

This is just the same as the usual form, except for the factor of $\beta_{Y}(y)$. We note that this implicitly assumes that our spans of span maps commute exactly - as in [39], we might also need to incorporate an explicit isomorphism up to which the diagram commutes. We note also that by (86), the maps $\left(M_{\sigma^{*} \alpha_{1}}\right)^{*}$ and $\left(M_{\tau^{*} \alpha_{2}}\right)^{*}$ are in fact equal, so again this natural isomorphism is an automorphism.

Combining these twisted variants on the ingredients of $\Lambda$, we have the following:

Definition 12 The 2-functor

$$
\Lambda^{U(1)}: \operatorname{Span}(\mathbf{G p d})^{U(1)} \rightarrow \mathbf{2 V e c t}
$$

consists of the following assignments.

- Objects: $\Lambda^{U(1)}\left(A, \theta_{A}\right)=\operatorname{Rep}\left(\mathbb{C}^{\theta_{A}}(A)\right)$

- Morphisms: To a span $\left(X, \alpha_{X}, s, t\right):\left(A, \theta_{A}\right) \rightarrow\left(B, \theta_{B}\right)$ define a 2-linear map:

$$
\Lambda^{U(1)}\left(X, \alpha_{X}, s, t\right)=t_{*} \circ\left(M_{\alpha_{X}}\right)^{*} \circ s^{*}
$$

where $M_{\alpha_{X}}: \mathbb{C}^{s^{*} \theta_{A}}(X) \rightarrow \mathbb{C}^{t^{*} \theta_{B}}(X)$ is the isomorphism of these groupoid algebras induced by multiplication by $\alpha_{X}$.

- 2-Morphisms: To a 2-morphism $\left(Y, \beta_{Y}, \sigma, \tau\right):\left(X_{1}, \alpha_{1}, s_{1}, t_{1}\right) \Rightarrow\left(X_{2}, \alpha_{2}, s_{2}, t_{2}\right)$ assign the natural transformation:

$$
\Lambda^{U(1)}\left(Y, \beta_{Y}, \sigma, \tau\right)=\epsilon_{L, \tau} \circ N_{\beta_{Y}} \circ \eta_{R, \sigma}:\left(t_{1}\right)_{*} \circ\left(M_{\alpha_{1}}\right)^{*} \circ s_{1}^{*} \Longrightarrow\left(t_{2}\right)_{*} \circ\left(M_{\alpha_{2}}\right)^{*} \circ s_{2}^{*}
$$

Remark 5 We have somewhat abused notation in order to write this in a balanced form. Strictly speaking, we have that:

$$
\eta_{R, \sigma}: \operatorname{Id}_{R e p\left(X_{1}, \alpha_{1}\right)} \Longrightarrow \sigma_{*} \circ \sigma *
$$

and similarly:

$$
\epsilon_{L, \tau}: \tau_{*} \circ \tau * \Longrightarrow \operatorname{Id}_{R e p\left(X_{2}, \alpha_{2}\right)}
$$


We have written them source and target, incorporating the multiplication operators $M_{\alpha_{i}}$ (and, though not written here, $M_{\sigma^{*} \alpha_{1}}$ and $M_{\tau^{*} \alpha_{2}}$ ). The point is just that

$$
\sigma_{*} \circ\left(M_{\sigma^{*} \alpha_{1}}\right)^{*} \circ \sigma^{*} \cong\left(M_{\alpha_{1}}\right)^{*} \circ \sigma_{*} \circ \sigma^{*}
$$

and similarly for $\tau$.

It may help to note that the cocycles at each level play somewhat independent roles, in this construction, though with our specific classical field theory $\mathcal{A}_{0}(-)_{G}^{\omega}$ they are closely related via transgression from $\omega$. This close connection may be an important part of the physical interpretation of this theory, and ensures we have a functor from $\mathbf{3 C o b}_{\mathbf{2}}$, but it is not essential to the "quantization functor" $\Lambda^{U(1)}$. The definition of $\Lambda^{U(1)}$ means we must have that $s^{*}\left(\theta_{A}\right)$ and $t^{*}\left(\theta_{B}\right)$ differ by the coboundary of $\alpha_{X}$ for the 2-linear map associated to a span to make sense (see the proof of the Theorem 4 below). However, $\alpha_{X}$ must be a cocycle, hence has coboundary 0 . So this is simply the requirement that $s^{*}\left(\theta_{A}\right) t^{*}\left(\theta_{B}\right)^{-1}=1$ in the definition of $\operatorname{Span}(\mathbf{G p d})^{U(1)}$. A similar remark applies to the 2-morphisms.

This means that the deep underlying relation between the $\theta, \alpha, \beta$ cocycles in our ETQFT is a property of the classical field theory, not a requirement of the quantization functor. Indeed, part of the point of this factorization is that the quantization functor contributes little to an understanding of the system: it essentially looks at a specific representation in 2Vect of structures already present in $\operatorname{Span}(\mathbf{G p d}){ }^{U(1)}$. To say this is a "representation" is to say precisely the following, which was implicitly stated in the above definition:

Theorem 4 The construction in Definition 12 determines a symmetric monoidal 2-functor

$$
\Lambda^{U(1)}: \operatorname{Span}(\mathbf{G p d}){ }^{U(1)} \rightarrow \mathbf{2 V e c t}
$$

This is the twisted version of [39, Thm. 5], though here we are also explicitly noting that the 2-functor is symmetric monoidal. Much of the proof is substantially the same as the untwisted case. We need to check several facts, so we will prove it as a series of lemmas, corresponding to lemmas and theorems shown in the untwisted case in [39]. The proofs are similar, so we will cite those at the appropriate place for brevity where there is significant overlap and show only the distinct new parts of the proofs.

Moreover, as in Theorem 3, in the interest of clarity we have not defined all of the specified structure morphisms which play the role for bicategories of properties of symmetric monoidal functors between categories. Because the construction of the 2 -functor provides natural choices for these morphisms, shall remark on what these choices are as we prove the necessary parts of this theorem.

To begin with, it is clear that $\operatorname{Rep}\left(C^{\theta_{A}}(A)\right)$ is a 2-vector space, since it is the category of representations of a finite dimensional complex algebra on complex vector spaces. Similarly, the functorial constructions for 1- and 2-morphisms ensure that we must obtain 2-linear maps and natural transformations. We must show that these assemble into a symmetric monoidal 2-functor. 
Lemma $5 \Lambda^{U(1)}$ preserves composition of 1-morphisms up to isomorphism.

Proof Note that this is the twisted version of [39, Thm. 3], which gives the corresponding isomorphism for the composites of spans in $\operatorname{Span}(\mathbf{G p d})$. This gives one of the structure maps for a weak 2-functor. As we shall see, we can inherit this structure from the untwisted version.

Suppose we are given two spans in $\operatorname{Span}(\mathbf{G p d})^{U(1)}$ :

$$
\left(X_{1}, \alpha_{1}, s_{1}, t_{1}\right):\left(A, \theta_{A}\right) \rightarrow\left(B, \theta_{B}\right)
$$

and

$$
\left(X_{2}, \alpha_{2}, s_{2}, t_{2}\right):\left(B, \theta_{B}\right) \rightarrow\left(C, \theta_{C}\right)
$$

Then the composite is:

$$
\left(X_{2} \circ X_{1}, \alpha_{2} \cdot \theta_{B} \cdot \alpha_{1}, s_{1} \circ S, t_{2} \circ T\right)
$$

The cocycle is that given in (89), and $\left(X_{2} \circ X_{1}, S, T\right)$ are the groupoid and maps in the weak pullback of the cospan $\left(B, t_{1}, s_{2}\right)$. It is shown in [39, Thm. 3], that there is an isomorphism

$$
\gamma: T_{*} \circ S^{*} \rightarrow\left(s_{2}\right)^{*} \circ\left(t_{1}\right)_{*}
$$

for the untwisted representation categories. It suffices to show a similar natural isomorphism between two functors:

$$
T_{*} \circ M_{\alpha_{2} \cdot \theta_{B} \cdot \alpha_{1}} \circ S^{*},\left(s_{2}\right)^{*}\left(t_{1}\right)_{*}: \operatorname{Rep}^{s_{1}^{*} \theta_{A}}\left[X_{1}\right] \rightarrow \operatorname{Rep}^{s_{2}^{*} \theta_{B}}\left[X_{2}\right]
$$

First, note that the induction and restriction functors for twisted representations are given by the usual formulas for modules of rings, and that the twisted groupoid algebras are characterized as a direct sum of twisted group algebras. These are the group algebras for automorphism groups of the objects in the $X_{i}$ and $B$, with multiplication twisted by the relevant 2-cocycles $\theta$. For clarity, we will use the following notation for these algebras that appear in the restriction and induction formulas:

$$
\begin{aligned}
\mathbb{A}_{x_{1}} & =\mathbb{C}^{s_{1}^{*} \theta_{A}}\left[\operatorname{Aut}\left(x_{1}\right)\right] \\
\mathbb{A}_{x_{2}} & =\mathbb{C}^{s_{2}^{*} \theta_{B}}\left[\operatorname{Aut}\left(x_{2}\right)\right] \\
\mathbb{A}_{t_{1}\left(x_{1}\right)} & =\mathbb{C}^{\theta_{B}}\left[\operatorname{Aut}\left(t_{1}\left(x_{1}\right)\right)\right] \\
\mathbb{A}_{x_{1}, x_{2}} & =\mathbb{C}^{\left(s_{1} \circ S\right)^{*} \theta_{A}}\left[\operatorname{Aut}\left(x_{1}\right) \times_{\operatorname{Aut}\left(t_{1}\left(x_{1}\right)\right)} \operatorname{Aut}\left(x_{2}\right)\right]
\end{aligned}
$$

Note that the cocycles mentioned are necessarily equal to others - for instance, $s_{1}^{*} \theta_{A}=$ $t_{1}^{*} \theta_{B}$, and so on.) 
So this natural transformation can be expressed at a stage $x_{1} \in X_{1}$ in terms of its action on a representation $\rho$. This is a linear map between spaces which are expressed as a direct sum over $x_{2} \in X_{2}$, and in each such summand we have:

$$
\gamma_{x_{1}}(F): \mathbb{A}_{x_{2}} \otimes_{\mathbb{A}_{x_{1}, x_{2}}} \rho\left(x_{1}\right) \rightarrow \mathbb{A}_{t_{1}\left(x_{1}\right)} \otimes_{\mathbb{A}_{x_{1}}} \rho\left(x_{1}\right)
$$

This is simply the twisted case of the usual formulas given as (91) and (92) in [39]. The isomorphism given there as (94) will still work in the twisted case. In the current notation, it acts in the following way. The algebra $\mathbb{A}_{x_{1}, x_{2}}$ decomposes as a direct sum over all $g \in \operatorname{Aut}\left(t_{1}\left(x_{1}\right)\right)$ (since it is a group algebra of a fibre product). In the summand associated to $g$ we define the isomorphism to act on the generators of $\mathbb{A}_{x_{2}} \otimes_{\mathbb{A}_{x_{1}, x_{2}}} \rho\left(x_{1}\right)$ by:

$$
(k \otimes v) \mapsto s_{2}(k) g^{-1} \otimes v
$$

which extends to the whole space. This is still well defined, though now uses the twisted multiplication in the group algebra $\mathbb{A}_{x_{2}}$. In particular, the underlying vector spaces are identical to the untwisted case. The proof that this is an isomorphism is substantially the same as in the untwisted case. The main difference is that the cocycle $\theta_{B}$ enters into the twisting of the multiplication in $\mathbb{A}_{x_{1}, x_{2}}$ and $\mathbb{A}_{t_{1}\left(x_{1}\right)}$. However, this twisting is compatible with the isomorphism $\gamma$ and the algebra maps induced by the homomorphisms $t_{1}$ and $s_{2}$ into $\left(B, \theta_{B}\right)$, so the proof is the same.

This then extends to an isomorphism between the two 2-linear maps

$$
\begin{array}{r}
\Lambda^{U(1)}\left(\left(X_{2}, \alpha_{2}, s_{2}, t_{2}\right) \circ\left(X_{1}, \alpha_{1}, s_{1}, t_{1}\right)\right) \\
=\left(T \circ t_{2}\right)_{*} \circ\left(M_{\alpha_{1} \cdot \theta_{B} \cdot \alpha_{2}}\right)^{*} \circ\left(S \circ s_{1}\right)^{*}
\end{array}
$$

and

$$
\begin{aligned}
& \left.\Lambda^{U(1)}\left(X_{2}, \alpha_{2}, s_{2}, t_{2}\right) \circ \Lambda^{U(1)}\left(X_{1}, \alpha_{1}, s_{1}, t_{1}\right)\right) \\
& =\left(t_{2}\right)_{*} \circ\left(M_{\alpha_{2}}\right)^{*} \circ\left(s_{2}\right)^{*} \circ\left(t_{1}\right)_{*} \circ\left(M_{\alpha_{1}}\right)^{*} \circ\left(s_{1}\right)^{*}
\end{aligned}
$$

Now we need the analogous fact for composition of 2-morphisms:

Lemma $6 \Lambda^{U(1)}$ preserves vertical composition of 2-morphisms strictly and horizontal composition of 2-morphisms up to the structure isomorphism of Lemma 5.

Proof This is the twisted analog of [39, Lemma 4] and [39, Lemma 5]. The proofs are just the same except that we now have the factors $N_{\beta}$ in (127). It thus suffices that the $N_{\beta}$ are multiplicative under both horizontal and vertical composition of 2-morphisms.

For vertical composition, suppose we are given 2-morphisms

$$
\left(Y, \beta_{Y}, \sigma, \tau\right):\left(X_{1}, \alpha_{1}, s_{1}, t_{1}\right) \Rightarrow\left(X_{2}, \alpha_{2}, s_{2}, t_{2}\right)
$$

and 


$$
\left(Y^{\prime}, \beta_{Y^{\prime}}, \sigma^{\prime}, \tau^{\prime}\right):\left(X_{2}, \alpha_{2}, s_{2}, t_{2}\right) \Rightarrow\left(X_{3}, \alpha_{3}, s_{3}, t_{3}\right)
$$

(Note that the current notation is different from that of [39], since here we use $(\sigma, \tau)$ instead of $(s, t)$, so that the structure maps for 2-morphisms are given by Greek letters and for 1-morphisms by Latin).

Then by (92), we have:

$$
\begin{aligned}
& \Lambda^{U(1)}\left(\left(Y^{\prime}, \beta_{Y^{\prime}}, \sigma^{\prime}, \tau^{\prime}\right) \circ\left(Y, \beta_{Y}, \sigma, \tau\right)\right) \\
& \quad=\Lambda^{U(1)}\left(Y^{\prime} \circ Y, \beta_{Y^{\prime}} \cdot \beta_{Y} \cdot \alpha_{2}, S \circ \sigma, T \circ \tau^{\prime}\right) \\
& =\epsilon_{L,(T \circ \tau)} \circ N_{\beta_{Y^{\prime}} \cdot \beta_{Y} \cdot \alpha_{2}} \circ \eta_{R,(S \circ \sigma)}
\end{aligned}
$$

The terms appearing here are defined in and following Definition 12. On the other hand, we have:

$$
\begin{aligned}
& \left.\Lambda^{U(1)}\left(Y^{\prime}, \beta_{Y^{\prime}}, \sigma^{\prime}, \tau^{\prime}\right) \circ \Lambda^{U(1)}\left(Y, \beta_{Y}, \sigma, \tau\right)\right) \\
& =\epsilon_{L,\left(\tau^{\prime}\right)} \circ N_{\beta_{Y^{\prime}}} \circ \eta_{R,\left(\sigma^{\prime}\right)} \circ \epsilon_{L,(\tau)} \circ N_{\beta_{Y}} \circ \eta_{R,(\sigma)}
\end{aligned}
$$

This composite agrees with (147) by a similar argument to that for 1-morphisms. Namely, $S$ and $T$ are the maps for the weak pullback $\left(\sigma^{\prime} \downarrow \tau\right)$, and these maps are compatible with twisted multiplication.

For horizontal composition, the proof is substantially the same as Lemma 5 of [39], except that factors of $\beta$ appear in the sums, and twisting by $\theta_{B}$ makes the maps of the pullback compatible with the twisted multiplication. The rest of the argument is substantially the same as for vertical composition.

So we have that composition of 2-morphisms is preserved.

Lemma 7 The 2-functor $\Lambda^{U(1)}$ is naturally equipped with the structure of a symmetric monoidal 2-functor of bicategories.

Proof Here we must define some extra structure maps. These arise naturally since tensor products essentially derive from $\times$ for groupoids.

First, (following [22]) there should be an adjoint equivalences relating the monoidal units:

$$
\iota: I \rightarrow \Lambda^{U(1)}(I)
$$

relating the two monoidal units. Explicitly, this is:

$$
\iota: \operatorname{Vect} \rightarrow \operatorname{Rep}(\mathbb{C})
$$

This is the obvious equivalence (indeed, isomorphism) of categories, since every vector space is automatically a representation of $\mathbb{C}=\mathbb{C}(1)$.

Then we need natural adjoint equivalences relating monoidal products:

$$
\chi 1,2: \Lambda^{U(1)}\left(A_{1}, \theta_{1}\right) \otimes \Lambda^{U(1)}\left(A_{2}, \theta_{2}\right) \rightarrow \Lambda^{U(1)}\left(\left(A_{1}, \theta_{1}\right) \otimes\left(A_{2}, \theta_{2}\right)\right)
$$


This comes from the natural inclusion:

$$
\mathbb{C}^{\theta_{1}}\left[A_{1}\right] \otimes \mathbb{C}^{\theta_{2}}\left[A_{2}\right] \rightarrow \mathbb{C}^{\theta_{1} \cdot \theta_{2}}\left[A_{1} \times A_{2}\right]
$$

taking $f \otimes g$ to the function $f g(a, b)=f(a) g(b)$. It is easy to check this is an algebra homomorphism since $\otimes$ just multiplies cocycles, and the twisted multiplication acts independently in each factor. Furthermore, in finite dimensions, this is clearly an isomorphism, since any function is a finite linear combination of delta-functions supported on individual morphisms of the $A_{i}$, or of their product.

The restriction and induction functors along this isomorphism are therefore an adjoint equivalence of categories.

This means that we have:

$$
\begin{aligned}
& \Lambda^{U(1)}\left(\left(A_{1}, \theta_{1}\right) \otimes\left(A_{2}, \theta_{2}\right)\right) \\
& \quad=\Lambda^{U(1)}\left(A_{1} \times A_{2}, \theta_{1} \cdot \theta_{2}\right) \\
& =\operatorname{Rep} \theta^{\theta_{1} \cdot \theta_{2}}\left[A_{1} \times A_{2}\right] \\
& =\operatorname{Rep}\left(\mathbb{C}^{\theta_{1} \cdot \theta_{2}}\left[A_{1} \times A_{2}\right]\right) \\
& \cong \operatorname{Rep}\left(\mathbb{C}^{\theta_{1}}\left[A_{1}\right] \otimes \mathbb{C}^{\theta_{2}}\left[A_{2}\right]\right)
\end{aligned}
$$

But this is generated by irreducible representations, and an irreducible representation of $\mathbb{C}^{\theta_{1}}\left[A_{1}\right] \otimes \mathbb{C}^{\theta_{2}}\left[A_{2}\right]$ is a tensor product of irreducible representations of $\mathbb{C}^{\theta_{1}}\left[A_{1}\right]$ and $\mathbb{C}^{\theta_{2}}\left[A_{2}\right]$. So this is isomorphic to:

$$
\begin{gathered}
\Lambda^{U(1)}\left(A_{1}, \theta_{1}\right) \otimes \Lambda^{U(1)}\left(A_{2}, \theta_{2}\right) \\
=\operatorname{Rep}^{\theta_{1}}\left[A_{1}\right] \otimes \operatorname{Rep}^{\theta_{2}}\left[A_{2}\right]
\end{gathered}
$$

with the tensor product the Deligne product of categories (for 2-vector spaces, this looks just like the usual tensor product of vector spaces in terms of generators, up to isomorphism).

There must also be coherent invertible modifications which give isomorphisms of certain composites of these natural adjoint equivalences (see [43]), and another relating $\chi$ and the braidings. These isomorphisms are quite natural, and a lengthy but straightforward check verifies the coherence conditions.

Taking the above three lemmas together we have the proof of Theorem 4. It is then immediate that this is an extension of our original 2-linearization 2-functor to the larger category, in the sense of the embedding noted in Corollary 1. That is:

Corollary 2 The restriction of $\Lambda^{U(1)}$ to $\operatorname{Span}(\mathbf{G p d}) \subset \operatorname{Span}(\mathbf{G p d})^{U(1)}$, is isomorphic with $\Lambda$.

Proof This is a straightforward consequence of applying the definitions with trivial cocycles, and the fact that the representation category of a finite groupoid is canonically isomorphic to that of its groupoid algebra. 
There is also a different special case, which is not immediately relevant to our ETQFT context, but which we will point out since it is immediate. This extends the fact that $\Lambda$ restricted to $\operatorname{Hom}(\mathbf{1}, \mathbf{1})$ reproduces Baez-Dolan groupoidification (shown in [39]). The new special case incorporates the $U(1)$-groupoids of [37]. Then a $U$ (1)-groupoid span (or "stuff operator" in the sense of [37]) is simply a nontrivial 2-morphism of $\operatorname{Span}(\mathbf{G p d})^{U(1)}$ in $\operatorname{Hom}((\mathbf{1}, 1),(\mathbf{1}, 1))$ between two 1-morphisms with trivial cocycles (moreover, [37] only considered the case where the central objects of these spans are always the groupoid of finite sets and bijections. This is not essentially finite, as in the present case, but provided we restrict to situations where all sums converge, the same ideas apply).

Finally, as in the untwisted case, the matrix representations of the 2-linear maps are straightforward to describe:

Proposition 6 Given a 1-morphism:

$$
(X, \alpha, s, t):\left(A, \theta_{A}\right) \rightarrow\left(B, \theta_{B}\right)
$$

the 2-linear map $\Lambda^{U(1)}(X, \alpha, s, t)$ has matrix representation whose components are:

$$
\Lambda^{U(1)}(X, \alpha, s, t)_{\rho, \phi}=H o m_{\operatorname{Rep}\left(\mathbb{C}^{*} \theta_{A X}\right)}\left(\left(s^{*} \rho\right),\left(\left(M_{\alpha}\right)^{*} t^{*} \phi\right)\right)
$$

Proof As in the untwisted case, this uses Frobenius reciprocity, in this case for representations of algebras. The operation of pulling back a representation $\phi$ of $\mathbb{C}^{\theta_{B}}[B]$ ) is adjoint to the operation of pushing-forward a representation of $\mathbb{C}^{t^{*} \theta_{B}}[X]$.

As in the untwisted case, the $\Lambda^{U(1)}(X, \alpha, s, t)$ takes a $\theta_{A}$-twisted representation of $A$, and pulls back to $X$, then pushes forward to $B$. The difference is that we apply the map $M_{\alpha}$ between these steps. By Frobenius reciprocity, we have the intertwiner space:

$$
\begin{aligned}
& \operatorname{Hom}_{\operatorname{Rep}\left(\mathbb{C}^{\theta} B[B]\right)}\left(t_{*} \circ M_{\alpha}^{*} \circ s^{*} \rho, \phi\right) \\
& =\operatorname{Hom}_{\operatorname{Rep}\left(\mathbb{C}^{\left.*^{*} \theta_{B}[X]\right)}\right.}\left(M_{\alpha}^{*} \circ s^{*} \rho, t^{*} \phi\right) \\
& =\operatorname{Hom}_{\operatorname{Rep}\left(\mathbb{C}^{\left.{ }^{*} \theta_{A}[X]\right)}\right.}\left(s^{*} \rho,\left(M_{\alpha}\right)_{*} \circ t^{*} \phi\right)
\end{aligned}
$$

So given irreducible twisted representations $\rho$ and $\phi$ of $A$ and $B$, (i.e. irreducible representations of the twisted groupoid algebras), we find a component in a matrix for a 2-linear map as an intertwiner space between the pulled-back representations $s^{*} \rho$ and $t^{*} \phi$. These are twisted representations of $\mathrm{X}$, but a priori we have that $s^{*} \rho$ is twisted by the cocycle $s^{*}\left(\theta_{A}\right)$, and $t^{*} \phi$ is twisted by $t^{*}\left(\theta_{B}\right)$.

We note that in principle, given two representations twisted by different cocycles, we would take the vector space of global sections of:

$$
\left(s^{\bar{*}} \rho\right) \otimes\left(t^{*} \phi\right)
$$


This is a vector bundle on the objects of $X$, and when the cocycles coincide, it corresponds to the usual hom space. The "bar" means we take the dual representation of $s^{*} \rho$, which is a $\left(s^{*} \theta_{A}\right)^{-1}$-twisted rep of X, so the tensor product (159) is a $\left(s^{*} \theta_{A}\right)^{-1} \times$ $\left(t^{*} \theta_{B}\right)$-twisted representation of $\mathrm{X}$.

This suggests that a further generalization of our $\operatorname{Span}(\mathbf{G p d})^{U(1)}$ may be possible in which the condition (84) can be weakened. We might only require the $\alpha$ be a cochain, and that $s^{*} \theta_{A}$ and $t^{*} \theta_{B}$ should differ by the coboundary of a cochain $\alpha$. This would ensure that $M_{\alpha}$ still induces an algebra isomorphism, but not necessarily an automorphism. If the condition were even weaker, the spaces of sections (159) would not correspond to intertwiner spaces. A similar generalization should be possible for condition (86), so that the coboundary of $\beta_{Y}$ gives the difference between pullbacks of $\alpha_{1}$ and $\alpha_{2}$.

This generalization, however, is not necessary for our construction of this ETQFT, so we will not consider it further here.

\subsection{Twisted ETQFT}

Finally, our main result asserts that the DW model can be understood as factorized into the classical field theory and the 2-linearization "quantization functor" we have just defined.

The theory itself, as described originally by Dijkgraaf and Witten [13], is given in more explicit detail by Freed and Quinn [17], particularly in the situation of manifolds with boundary, which is the case where an ETQFT is most appropriate. This is the description to which we will refer here when speaking of the DW model. In particular, much of the description we use is in [17, Sec. 4] which describes its construction as a modular functor.

We will describe how it is derived from the 2-functor we have given as our ETQFT.

Theorem 5 Given a finite gauge group $G$ and 3-cocycle $\omega \in Z^{3}(B G, U(1))$, the symmetric monoidal 2-functor

$$
Z_{G}^{\omega}=\Lambda^{U(1)} \circ \mathcal{A}_{0}(-)_{G}^{\omega}: \mathbf{3} \mathbf{C o b}_{2} \rightarrow \mathbf{2 V e c t}
$$

reproduces the DW model with twisting cocycle $\omega$.

Proof First, we note that the DW model as described in [17] assigns a Hilbert space $E(Y)$ to each 2D manifold $Y$ with boundary. We regard think of this manifold as a 2D cobordism between 1D boundary components, and describe the correspondence between $E(Y)$ and the 2-linear map assigned by the $Z_{G}$ given by our construction. Horizontal composition of 1- and 2-morphisms corresponds to the trace over a tensor product of the data associated to a boundary in which incoming and outgoing boundary components are distinguished by orientation.

So, to the 1D boundary in such a case, the DW model assigns a collection of labels. These are irreducible representations of certain algebras. Due to the monoidal 
structure, this reduces to the case of the algebra assigned to a circle, which is:

$$
A^{*}=\bigoplus_{[T]} L_{[T]}^{*}
$$

This is a direct sum over [ $T$ ], the distinct conjugacy classes in $G$, which is to say, the isomorphism classes of objects of $\mathcal{A}_{0}\left(S^{1}\right)_{G}$. The algebras $L^{*}$ and connecting isomorphisms between them form a line bundle over the space of [T], which is classified by a cohomology class given by the transgression of the form there called $\hat{\alpha}$, and here $\omega$ in $H^{3}(B G, \mathbb{R} / \mathbb{Z})$ (this is [17, Proposition 3.14]).

So the cocycles $\beta_{\mathcal{A}_{0}(M)_{G}}$ are as we expect for a 3-dimensional cobordism $M$. We have summarized these functorial properties by the observation of 5.1 that the assignment of cocycles is an HQFT.

Now the algebra structure of $L_{[T]}^{*}$ is such that its unit vectors form a central extension of the centralizer of [T] (that is, Aut ([T]) in the sense of the groupoid $\left.\mathcal{A}_{0}\left(S^{1}\right)_{G}\right)$. The central extension is classified by the cocycle just mentioned. In our terminology, this says precisely that $L_{[T]}^{*}$ itself is the summand of $\mathbb{C}^{\tau} S^{1}{ }^{(\omega)}\left[\mathcal{A}_{0}\left(s^{1}\right)_{G}\right]$ associated to $[T]$. Thus,

$$
A^{*} \cong \mathbb{C}^{\tau} S^{1(\omega)}\left[\mathcal{A}_{0}\left(S^{1}\right)_{G}\right]
$$

But our $Z_{G}^{\omega}$ assigns the circle the representation category of this algebra, which recovers the label set assigned by the DW model.

Next, the DW model assigns a Hilbert space to each manifold with boundary $Y$ (in the following we use the notation of [17, Section 3]). We will understand this to be a cobordism relating its boundary components. Thus, this Hilbert space is to be understood as a 2-linear map. As a Hilbert space, the $E(Y)$ are given as:

$$
E(Y)=L^{2}\left(\overline{\mathcal{C}_{Y}^{\prime}}, \overline{\mathcal{L}_{Y}}\right)
$$

That is, it is the space of (square-integrable, which condition is vacuous in the finite case) sections of a certain line bundle $\mathcal{L}_{Y}$ over the space $\mathcal{C}_{Y}^{\prime}$ of flat connections (i.e. bundles with flat connections) on $Y$, which we would describe as the space of objects of $\mathcal{A}_{0}(Y)_{G}$. This bundle assigns a 1-dimensional space to each such object, and to each homotopy of the classifying maps of these flat bundles (i.e. to each morphism $f$ of $\mathcal{A}_{0}(Y)_{G}$ ) an isomorphism of these lines, given by (3.4) of [17]. This isomorphism incorporates a factor which comes from an integral of $\hat{\alpha}$, or in our terms $\omega$. This factor is just the value of $\tau_{Y}(\omega)$ on $f$.

A decomposition of the space of sections $E(Y)$ as a direct sum of components is given in [17, sec. 4$]$ as:

$$
E(Y) \cong \bigoplus_{\lambda} E(Y, \lambda) \otimes E_{\lambda}
$$

Here, the $\lambda$ run over all labels for the boundary: this is a product of labels $\lambda=\left(\lambda_{i}\right)_{i}$ over all boundary components $(\partial Y)_{i}$. The representations $E_{\lambda}$ associated to the whole 
boundary are therefore of the form $\otimes_{i} E_{\lambda_{i}}$. By the duality of Hom and $\otimes$, these are isomorphic to the intertwiner spaces given in $(157)$. The $E(Y, \lambda)$ give the multiplicities of these representations.

The above decomposition amounts to treating $E(Y)$ as a module for $A^{\star}$ for the algebra associated to $\partial Y$, which acts on the $E_{\lambda}$, or rather as a bimodule for the algebras $A^{\star}$ for the source and target objects (taking the conjugate algebra when changing orientation, hence turning a left action into a right action). Frobenius reciprocity then ensures that taking a tensor product with this bimodule will act as multiplying by the matrix (157). This gives an interpretation of $E(Y)$ as the 2-linear map $Z_{G}^{\omega}(Y)$.

The DW model then assigns a map between these Hilbert space $E(Y)$ for each cobordism between manifolds $Y$ and $Y^{\prime}$. We further note that the inner product on this space, as a space of sections, is twisted by the cocycle $\alpha$, which is accomplished precisely by the inclusion of the map $M_{\alpha}$ in our construction of the 2-linear map $Z_{G}^{\omega}(Y)$.

Finally we check that $Z_{G}^{\omega}=\Lambda^{U(1)} \circ \mathcal{A}_{0}(-)_{G}^{\omega}$ gives the data of the twisted DW model for 2-morphisms $M$ of $\mathbf{3} \mathbf{C o b}_{2}$, which are understood as 3-dimensional cobordisms of manifolds with boundary. In [17] they are described as manifolds with corners.

Part of this proof is substantially the same as that of Theorem 2, which shows in the untwisted case with empty boundary that our formula reproduces the (unnumbered) formula directly following $[17,5.14]$. That formula uses the "mass" of a connection on (there described as a "representation" of $\pi_{1}(M)$ into the gauge group - though they denote the manifold by $Y$ ), which is just the groupoid cardinality $\frac{1}{A u t([A])}$ for a class $[A]$ of connections (denoted there by $\gamma$ ), as in our formula. This gives the measure used in the integrals over the space of connections, as we expect.

Finally, the explicit calculations of amplitudes in [17] are generally contractions of the 2-linear maps we obtain. Moreover, they are converted to amplitudes from linear operators between representation spaces by converting representations to characters, taking the trace. Thus, since the trace of the identity for a representation $\rho$ is $\operatorname{dim}(\rho)$, the formulae there contain factors of $\operatorname{dim}\left(E_{\lambda}\right)$, where $E_{\lambda}$ is the representation space for a representation on the whole boundary (for us, the tensor product of the representations determining a given component of the natural transformation). So finally the computations of amplitudes such as $[17,5.4]$ for the torus are precisely the result of applying this procedure to the natural transformations from $\Lambda^{U(1)}$.

We conclude that the DW model for manifolds with corners as presented in [17] can be recovered from our $Z_{G}^{\omega}$.

\section{Conclusion}

In this paper, our goal has been to give a concrete description of the quantization functor which plays a role in the DW model. This is consistent with the program of Freed-Hopkins-Lurie-Teleman [16], in which topological quantum field theories are described in terms of a factorization into two parts. The first part, the classical field theory, takes values in groupoids. The second part assigns algebraic data to groupoids - in particular, $k$-vector spaces, or indeed $k$-algebras in an appropriate sense, to the groupoids associated to codimension- $k$ manifolds. 
The point is that the groupoids represent the moduli space for the field configurations of the classical theory. As we have seen, the full functor, to describe the DW model in its complete form, must incorporate the effect of data from groupoid cohomology.

One purpose of studying the quantization functor separately is that we hope to gain some understanding of the nature of the quantization process. Quantization is well-studied in the situation of a process (in good situations, a functor) taking classical configuration spaces to quantum Hilbert spaces. The higher-categorical $k$-vector spaces are less commonly used in the physical context and ETQFT gives a sufficiently simple, yet nontrivial, setting in which to study this aspect of quantization. What our functors $\Lambda$ and its twisted version $\Lambda^{U(1)}$ illustrate is that this process can be described in terms of a simple, quite universal process once we understand the category $\operatorname{Span}(\mathbf{G p d})$, or its twisted version $\operatorname{Span}(\mathbf{G p d})^{U(1)}$.

In particular $\Lambda^{U(1)}$ is an extension of the very natural "2-linearization" process $\Lambda$, which is entirely canonical. Groupoids are taken to their representation categories. The morphisms (spans) are taken to 2-linear maps constructed naturally from induction and restriction functors. The 2-morphisms (spans of spans) are taken to natural transformations constructed naturally from the unit and counit for the adjunctions between these functors. This is an entirely canonical process generalizing the straightforward "pull" and "push" of functions through spans of sets which gives (natural number valued) matrix multiplication. Thus, the quantization functor is simply giving a canonical representation of Span $(\mathbf{G p d})$, which is then in some sense the fundamental setting.

One important fact in the untwisted case is that, if we take the representation category $\Lambda(A)$ to be concrete, with its natural "underlying vector space" functor into Vect, we have a Tannaka-Krein reconstruction theorem. That is, this 2-vector space (and the forgetful functor into Vect) allows the groupoids (objects of $\operatorname{Span}(\mathbf{G p d})$ ) to be reconstructed completely. At the level of morphisms, and particularly 2-morphisms, however, we do lose information. This is easy to see in the special case of $\operatorname{Hom}(\mathbf{1}, \mathbf{1})$, where $\Lambda^{U(1)}$ restricts to give groupoidification. Here, spans of groupoids, as morphisms, are taken to linear maps whose components detect only groupoid cardinalities of spans. This does not determine the groupoids up to isomorphism. So in particular, the quantization functor is not faithful, and forgets information about the classical category as part of the "sum over histories" which defines the 2-morphisms.

The motivation for using $\operatorname{Span}(\mathbf{G p d})$ and its twisted extension is how it reflects physically important aspects of the quantum field theory. The objects are groupoids because the moduli problem for gauge theory, like many other geometric structures, has symmetries which are not seen in a topological space of field configurations. The quantization functor, our $\Lambda^{U(1)}$, is able to retain this information about symmetry, since it assigns the representation category to such groupoids. This characterizes them up to Morita equivalence. In general, systems whose configuration spaces are represented by Morita-equivalent groupoids are "physically indistinguishable".

However, rather than working in the bicategory Gpd, we expand it to consider Span(Gpd). We have noted some work [25,28] on such span categories generally. One important fact is that in general $\operatorname{Span}(\mathbf{C})$ for a (1-)category is a universal (bi)category containing a copy of $\mathbf{C}$, for which every morphism has a (two-sided) adjoint. In the case C is a bicategory, such as Gpd, our construction also gives adjoints for 2-morphisms (in [25], one gets a monoidal tricategory, which we have made into a bicategory by taking 
2-morphisms as mere equivalence classes of spans of span maps). This construction of "adjoining adjoints" is somewhat analogous to localization, which forces morphisms to be invertible. Instead, we force morphisms to be adjointable. This is the key feature captured in $\operatorname{Span}(\mathbf{G p d})$, and is also a key characteristic of the linear and 2-linear category.

The physical significance of adjointability is that if a morphism describes a process by which a system evolves, its adjoint is the same process with the reversed timesense. In the ETQFT case, the cobordism category suggests that we should think of 2-morphisms as "time evolution" in this sense. The 1-morphisms then describe a space with boundary as linking its boundary components, and the adjoint simply reverses the sense of input and output boundary components.

In the twisted case, we must expand this setting to $\operatorname{Span}(\mathbf{G p d})^{U(1)}$, but this behaves much like Span $(\mathbf{G p d})$ except that the groupoids carry extra cocycle information. This information is the higher-categorical extension of the Lagrangian functional, which is simply the 0-cocycle associated to 2-morphisms. This fits the approach of [16], in which the cocycle $\omega$ on $B G$, and the gerbe it classifies, is taken to be the true physical setting for the action. The transgressions to the moduli spaces for connections on manifolds of different dimensions are then particular manifestations of this action.

In subsequent work, it may be of interest to consider whether this larger bicategory $\operatorname{Span}(\mathbf{G p d})^{U(1)}$, or perhaps a weaker variation, has some important universal properties analogous to those of $\operatorname{Span}(\mathbf{G p d})$. For now, it is sufficient to observe that it is the natural target for the classical field theory of the DW model, and likely other interesting toy physics models relevant to TQFT. A subsequent paper by the author with Derek Wise will consider an analogous construction with compact Lie groups and give an explicit construction of a generalization of $\Lambda$ and $\Lambda^{U(1)}$ which applies in the infinite setting.

Acknowledgments The author would like to thank the referees of earlier versions for important suggestions, as well as John Baez, Dan Christensen, Alex Hoffnung, Thomas Nikolaus, Mike Stay, Jamie Vicary, and Derek Wise for valuable discussions. This work was partially financed by Portuguese funds via the Fundacao para a Ciencia e a Tecnologia, through project number PTDC/MAT/101503/2008, New Geometry and Topology.

\section{References}

1. Adem, A., Cohen, F. R., Torres-Giese, E.: Commuting elements, simplicial spaces, and filtrations of classifying saces. arXiv:0901.0137 (2009)

2. Atiyah, M.: Topological Quantum Field Theory. Cambridge University Press, Cambridge (1990)

3. Baez, J.: Higher-dimensional algebra II: 2-hilbert spaces. Adv. Math. 127, 125-189 (1997). arXiv:qalg/9609018

4. Baez, J., Dolan, J.: Higher-dimensional algebra and topological quantum field theory. J. Math. Phys. 36, 6073-6105 (1995). arXiv:q-alg/9503002

5. Baez, J.C., Hoffnung, A.E., Walker, C.D.: Groupoidification made easy. arXiv:0812.4864 (2013, preprint)

6. Baez, J.C., Hoffnung, A.E., Walker, C.D.: Higher dimensional algebra vii: groupoidification. arXiv:0908.4305 (2009)

7. Bartlett, B.: Categorical aspects of topological quantum field theories. Master's thesis, Utrecht University. arXiv:math/0512103 (2005) 
8. Benson, D.: Representations and cohomolgy I: basic representation theory of finite groups and associative algebras. No. 30 in Cambridge Studies in Advanced Mathematics. Cambridge University Press, Cambridge (1995)

9. Brown, R.: Groupoids and Topology. Booksurge (2006)

10. Cheng, E., Lauda, A.: Higher-dimensional categories: an illustrated guidebook. http://www.dpmms. cam.ac.uk/elgc2/guidebook/ (preprint)

11. Chorny, B.: Brown representability for space-valued functors. http://arxiv.org/abs/0707.0904 (2007)

12. Dawson, R.J.M., Paré, R., Pronk, D.A.: Universal properties of span. Theory Appl. Categ. 13(4), 61-85 (2004)

13. Dijkgraaf, R., Witten, E.: Topological gauge theory and group cohomology. Commun. Math. Phys. 129 (1990). http://math.ucr.edu/home/baez/qg-winter2005/group.pdf

14. Dubuc, E.J.: Kan extensions in enriched category theory. Lecture Notes in Mathematics, vol. 145. Springer, Berlin (1970)

15. Freed, D.S.: Higher algebraic structures and quantization. arXiv:hep-th/9212115 (1993)

16. Freed, D.S., Hopkins, M.J., Lurie, J., Teleman, C.: Topological quantum field theories from compact Lie groups. arXiv:0905.0731 (2009)

17. Freed, D.S., Quinn, F.: Chern simons theory with finite gauge group. Commun. Math. Phys. 156 (1993)

18. Gordon, R., Power, A., Street, R.: Coherence of Tricategories. American Mathematical Society, Memoirs of the American Mathematical Society. American Mathematical Society (1995)

19. Grandis, M.: Higher cospans and weak cubical categories (cospans in algebraic topology i). Theory Appl. Categ. 18(12), 321-347 (2007)

20. Grandis, M.: Cubical cospans and higher cobordisms (cospans in algebraic topology iii). J. Homotopy Relat. Struct. 3, 1 (2008)

21. Greenlees, J.: Rational mackey functors for compact Lie groups I. Proc. Lond. Math. Soc. 3(76), 549-578 (1998)

22. Gurski, N.: The Algebraic Theory of Tricategories. PhD thesis, University of Chicago, Chicago (2006)

23. Higgins, P.J.: Categories and Groupoids. No. 32 in Van Nostrand Reinhold Mathematical Studies. Van Nostrand Reinhold Company, London (1971)

24. Hoffnung, A.: The hecke bicategory. arXiv:1007.1931

25. Hoffnung, A.: Spans in 2-categories: a one-object tetracategory. Unpublished draft at http://mysite. science.uottawa.ca/hoffnung/papers_files/spans.pdf

26. Kapranov, M., Voevodsky, V.: 2-Categories and Zamolodchikov tetrahedron equations. Proc. Symp. Pure Math. 56(Part 2), 177-260 (1994)

27. Kashiwara, M., Schapira, P.: Categories and Sheaves. Grundlehren der mathematischen Wissenschaften, vol. 332. Springer, Berlin (2000)

28. Kenney, T., Pronk, D.: The span profunctor and generalised span constructions. Unpublished draft

29. Kerler, T., Lyubashenko, V.V.: Non-semisimple Topological Quantum Field Theories for 3-Manifolds with Corners. Lecture Notes in Mathematics, vol. 1765. Springer, Berlin (2001)

30. Lack, S.: A 2-categories companion. In: Baez, J.C., May, J. (eds.) Towards Higher Categories, No. 152 in The IMA Volumes in Mathematics and its Applications. Springer, Berlin, pp. 105-191 (2010)

31. Lauda, A.D.: Frobenius algebras and ambidextrous adjunctions. Theory Appl. Categ. 16(4), 84-122 (2006)

32. Lawrence, R.: Triangulation, categories and extended field theories. In: Baadhio, R., Kaufman, L. (eds.) Quantum Topology, pp. 191-208. World Scientific, Singapore (1993)

33. Lurie, J.: On the classification of topological field theories. arXiv:math.CT/0905.0465

34. MacLane, S., Moerdijk, I.: Sheaves in Geometry and Logic: A First Introduction to Topos Theory. Universitext. Springer, New York (1992)

35. McCrudden, P.: Balanced coalgebroids. Theory Appl. Categ. 7(6), 71-147 (2000)

36. Moerdijk, I.: Étale groupoids, derived categories, and operations. In: Groupoids in Analysis, Geometry, and Physics. American Mathematical Society, Providence, Contemp. Math., vol. 282, pp. 101-114 (2001)

37. Morton, J.C.: Categorified algebra and quantum mechanics. Theory and Applications of Categories, vol. 16, 785-854 (2006). http://www.tac.mta.ca/tac/volumes/16/29/16-29.pdf

38. Morton, J.C.: Double bicategories and double cospans. J. Homotopy Relat. Struct. 4, 1, 389-428. arXiv:math/0611930 (2009, preprint)

39. Morton, J.C.: 2-vector spaces and groupoids. Applied Categorical Structures. arXiv:0810.2361. doi:10. 1007/s10485-010-9225-0 (2010, preprint, final version) 
40. Panchadcharam, E.: Categories of Mackey Functors. PhD thesis, Macquarie University (2006)

41. Picken, R.F.: Tqft's and gerbes. Alg. Geom. Topol. 4, 243-272 (2004)

42. Porter, T.: The crossed menagerie: an introduction to crossed gadgetry and cohomology in algebra and topology. http://ncatlab.org/nlab/files/menagerie10a.pdf

43. Schommer-Pries, C.: The classification of two-dimensional extended topological field theories. $\mathrm{PhD}$ thesis, University of California, Berkeley. arXiv:1112.0040 (2009)

44. Stay, M.: Compact closed bicategories. arXiv:1301.1053

45. Sternberg, S.: Group Theory and Physics. Cambridge University Press, Cambridge (1994)

46. Thurston, W.: Three-Dimensional Geometry and Topology. Princeton University Press, Princeton (1997)

47. Turaev, V.: Quantum Invariants of Knots and 3-Manifolds. No. 18 in De Gruyter Studies in Mathematics. Walter de Gruyter (1994)

48. Turaev, V.: Homotopy Quantum Field Theory. No. 10 in Tracts in Mathematics. European Mathematical Society (2010)

49. Willerton, S.: The twisted Drinfeld double of a finite group via gerbes and finite groupoids. Algebr. Geom. Topol. 8(3), 1419-1457 (2008)

50. Yetter, D.: Categorical linear algebra - a setting for questions from physics and low-dimensional topology. Kansas State University. http://math.ucr.edu/home/baez/yetter.pdf (2013, preprint)

51. Yetter, D.: Topological quantum field theories associated to finite groups and crossed $G$-sets. J. Knot Theory Ramifications 1(1), 120 (1992) 